\title{
Three One-factor Processes for Option Pricing with a Mean-reverting Underlying: The case of VIX
}

\author{
Bo Zhao ${ }^{\mathrm{a}, *}$, Cheng Yan $^{\mathrm{b}, *}$, Stewart Hodges ${ }^{\mathrm{c}}$ \\ ${ }^{a}$ Fucius Capital Hedge Fund London, E14 3AP, UK. \\ ${ }^{b}$ Durham University Business School, Millhill Lane, Durham, DH1 3LB, UK. \\ 'Sir John Cass Business School, London, EC1Y 8TZ, UK.
}

\begin{abstract}
We challenge the two most prominent one-factor mean-reverting models for variance/volatility indices and propose the Inhomogeneous Geometric Brownian Motion (IGBM) process to price VIX options. We study the roles of the equilibrium level, speed of reversion, volatility and expiration date in the pricing of VIX options and obtain analytic solutions for perpetual American options as well as some Greeks. We price the finite-lived American options using their transformed process to build a binomial tree. We also derive the closed-form Mean FirstPassage Time (MFPT) for perpetual American options and find a long optimal exercising time. Other things being equal, the European ones are cheaper than their American counterparts, which reflects an early exercise premium.

Keywords: Volatility derivative; American option; Mean-reverting process; Implied volatility
\end{abstract}

\footnotetext{
${ }^{\star 2}$ We are grateful to Carol Alexander, Peter Carr, Ding Chen, Yaozhong Hu, Andreas Kaeck, Andreas Kyprianou, Oliver Linton, Goran Peskir, Nashwa Saleh, Radu Tunaru, and participants in the 4th general AMaMeF conference at Aalesund, the spectral and cubature methods in finance and econometrics workshop at Leicester, as well as workshops in Cass Business School, Durham University Business School, Sussex University and Warwick Business School for helpful comments. We alone are responsible for all errors.

${ }^{*}$ Co-corresponding authors: Cheng Yan, email: cheng.yan@durham.ac.uk; Bo Zhao, email: 409541076@qq.com.
}

Preprint submitted to Financial Review

April 6, 2018 
JEL Classification: G13, C02 
'VIX options and futures are among the most actively traded contracts at CBOE and CFE ... Although these new assets certainly offer additional investment and hedging opportunities for financial market participants, their correct use requires reliable valuation models that adequately capture the features of the underlying volatility index.'

Mencía and Sentana (2013)

\section{Introduction}

Unlike many financial products, theoretical considerations of the pricing of VIX derivatives appear in academia long before their actual introduction. The concept of variance trading, such as variance swap contracts, appears first in Neuberger (1990, 1994). After further developments in the literature (e.g., Carr and Madan (1998), Demeterfi, Derman, and Kamal (1999); Demeterfi, Derman, Kamal, and Zou (1999)), many volatility derivatives became actively traded in financial markets. These volatility instruments do not only allow investors to hedge the volatility risk of their equity market positions, but also provide opportunities for them to bet on the implied volatility speculatively. Although so far only European-type volatility derivatives are available for trading in the industry, examining the characteristics and behaviors of American options written on VIX (hereafter, VIX options) are interesting for two main reasons. On the one hand, as opposed to standard European-type contracts where options can only be exercised at expiration dates (hereafter, expiration, in short), American options can be exercised at any time before expiration. Consequently, the whole prospective path is important and the optimal exercise boundary is crucial to pricing. On the other hand, with a mean-reverting underlying VIX index, the characteristics of VIX options are quite different from the ones of the conventional options with a Geometric Brownian Motion underlying asset.

When pricing a European option and a futures contract with the same underlying asset and the same expiration, we can simply model the futures price as following a martingale (i.e., with no drift) under the risk-neutral measure by using the bank account as the standard measure (i.e., numeraire). Similarly, when pricing an American option, we also have a conve- 
nient martingale for the process of the underlying asset. However, an American option with an underlying index (i.e., the VIX index) which is not a tradable asset is more complicated. Researchers have to take into account the varying drift and the diffusion component of the process, both of which affect the option value. More specifically, when the drift is larger than the riskless return, the situation can be regarded as if the asset gains a convenience yield. It is optimal to wait to exercise American call options. In contrast, when the drift is smaller than the riskless return, the situation can be regarded as if the asset pays a dividend, and it is worthwhile to exercise American call options early. However, the convenience yield (or apparent dividend rate) now varies stochastically depending on the level of the underlying asset/index. The presence of mean-reversion can switch the convenience yield to a dividend rate and back again. Hence, both American call options and put VIX options are different from their European counterparts.

Earlier models for VIX derivatives include the Black-Scholes Geometric Brownian Motion (GBM) type model by Whaley (1993) $)^{1}$, the Feller (1951) process by Grünbichler and Longstaff $(1996)^{2}$, and the Geometric Ornstein-Uhlenbeck (GOU) by Detemple and Osakwe (2000) ${ }^{3}$. In the recent literature, Mencía and Sentana (2013) empirically analyze the pricing performance of VIX derivatives under these two most prominent mean-reverting processes (as well as as well as their variants such as time-varying central tendency, volatility jumps, and stochastic

\footnotetext{
${ }^{1}$ Hence, it implicitly assumes that the VIX does not mean-revert at all, which is understandable given the fact that earlier literature in the 1980s and 1990s (see, e.g., Pagan and Schwert (1990); Schwert (1990)) finds a unit root in stock volatility when the data span the 1930s. However, recent literature identifies strong evidence and convincingly argues that the VIX has become mean-reversing, especially during and after the late 2000s Global Financial Crisis (GFC) (see, e.g., Schwert (2011), which excludes the GBM model as a candidate for our analysis.). ${ }^{2}$ The Feller (1951) process is also called the Heston (1993) process, which is widely used in finance. For instance, Lin and Chang (2009) assume that the S\&P 500 index, rather than the VIX dynamics, follows the Heston (1993) process, which is usually called the consistent pricing framework (see, e.g., Egloff, Leippold, and Wu (2010), Zhu and Lian (2012)).

${ }^{3}$ They price options on instantaneous variance.
} 
volatility of variance) proposed so far for the VIX 4 . Mencía and Sentana (2013) documents that the arguably "success" and popularity of both the Feller and the GOU process, as well as their variants, are because the sample periods only cover a relatively long and quite bull market, which ends at the GFC. Despite these glaring deficiencies of these two fundamental processes, Mencía and Sentana (2013) did not propose an alternative one-factor process to replace them.

Neither the Feller model nor the GOU model is able to capture the most relevant stylized fact of the VIX market, which is a positive skew of the implied volatility, as documented by Wang and Daigler (2011) and Mencía and Sentana (2013). The transition density functions of both the GBM and the GOU are log-normal, generating a constant implied volatility across strikes. The Heston process and its variations are widely adopted in modeling stochastic volatility of the VIX and particularly of the equity index dynamics, generating a negative skew of the implied volatility. Beyond the Heston process and these processes with log-normal distributions, the lack of analytical solution to option pricing has hindered progress in the development of continuous time volatility modeling. Other than the GOU and Feller models, we also consider the Inhomogeneous Geometric Brownian Motion (IGBM) process (aka the GARCH diffusion model for the variance rather than volatility in Nelson (1990); Lewis (2000)), whose steadystate distribution displays a heavy right tail capturing the positive skew of the implied volatility.

A couple of reasons motivate our choice of IGBM model rather than the GOU and Feller model. On the one hand, the Black-Scholes implied volatility of VIX options is positively skewed, while neither the Feller process nor the process with log-normal distributions can capture this most relevant stylized fact of the VIX market. Traditionally, the literature on equity index option pricing, address the deficiencies of the one-factor stochastic volatility models by adding a jump proces $5^{5}$ or more factors (e.g., Huang and Wu (2004); Christoffersen,

\footnotetext{
${ }^{4}$ They alternatively call the Feller process and the GOU process, the SQuare Root (SQR) process and the Log-normal Ornstein-Uhlenbeck (LOU) process, respectively.

${ }^{5}$ See, e.g., Bates (1996, 2000); Bakshi, Cao, and Chen (1997); Andersen, Benzoni, and Lund (2002); Pan
} 
Jacobs, Ornthanalai, and Wang (2008); Christoffersen, Heston, and Jacobs (2009); Branger, Kraftschik, and Völkert (2016) $)^{6}$. Similarly, Branger, Kraftschik, and Völkert (2016) show that both adding positive sized jumps to the variance process and introducing a stochastic volatility of variance factor which positively correlates to the variance process can achieve positive implied skews. This study does not question the usefulness of this approach. Alternatively, we take a much simpler approach to capture positive skews, than do these jump or multi-factor models. The simplicity is particularly necessary for this issue as it allows us to derive the closeform solution for American-style VIX options and compare them with their European-style counterparts. On the other hand, Aït-Sahalia and Kimmel (2007) find that the coefficient $\beta$ in the CEV model (a more general model covers both the GOU $(\beta=0)$ and the $\operatorname{IGBM}(\beta=1))$ is estimated by 0.94 using the daily VIX., which also motivates our choice of IGBM model rather than the GOU model.

We investigate the roles of the equilibrium level, the speed of reversion, the volatility and expiration date in the valuation, with perpetual options as a limiting case. Our closed-form expressions for the perpetual American case provide a straightforward way to examine the roles played by the equilibrium mean level, the speed of reversion, and the volatility. The optimal exercising boundary is unique to price American options. Typically, investors are interested in knowing when the option will be exercised. To measure the optimal exercising time, we compute its Mean First-Passage Time (MFPT). The MFPT plays an important role in the valuation of perpetual (real) options: e.g., Mauer and Ott (1995) use the MFPT to estimate the optimal time to undertake a replacement investment. Due to the nature of volatility, we find that the MFPT is quite long in exercising volatility options. We then consider three dif-

(2002); Chernov et al. (2003); Eraker, Johannes, and Polson (2003); Eraker (2004); Carr and Wu (2004); Huang and Wu (2004); Broadie, Chernov, and Johannes (2007); Santa-Clara and Yan (2010).

${ }^{6}$ It is worthwhile to note that, as pointed by (Christoffersen, Heston, and Jacobs, 2009, p. 1915-1916), "As a consequence, many papers use a single-factor stochastic volatility model as the starting point for more complex models...multifactor models have not yet become more popular in the option valuation literature". 
ferent mean-reverting processes which have very different properties and discuss the impact of different mean-reverting dynamics for the pricing of American VIX options. We find that American option values are more sensitive to the choice of the underlying process than their European counterparts, which underscores the importance of our proposed IGBM for American VIX options.

Our paper directly draws upon studies that seek to price VIX options, and is also linked, albeit more indirectly, with studies investigating the pricing of options with a mean-reverting underlying index. A concurrent purely theoretical paper, Detemple and Kitapbayev (2018), captures this stylized fact using a mixture of the generalized $3 / 2$ and $1 / 2$ models, but sacrifices model simplicity and parsimony. Our model can be used as a new starting point for the pricing of VIX options and other options with a mean-reverting underlying asset/index, by adding volatility jump components (see, e.g., Wu (2011)), volatility on volatility (see, e.g., Branger, Kraftschik, and Völkert (2016)), and more factors (see, e.g., Christoffersen, Heston, and Jacobs (2009)).

This paper also contributes to the literature on real options, as the valuation of investment in an irreversible project is pricing the perpetual American options. We assume the project value follows a stochastic process, and $K$ denotes the initial cost in implementing the project. The investment decision problem in capital budgeting is equivalent to pricing an American perpetual (call) option with strike K. For instance, Bhattacharya (1978), Dixit and Pindyck (1994), Metcalf and Hassett (1995), Sarkar (2003) examine the project value under the mean-reverting assumption. The paper complements these studies by providing additional analytical insights about the impact of the speed of reversion, the equilibrium mean level, and the volatility on the valuation of irreversible projects.

\section{Inhomogeneous Geometric Brownian Motion Process}

According to the extant literature (see, e.g., Wang and Daigler (2011) and Mencía and Sentana (2013)), the two most well-known mean-reverting processes to describe the VIX dy- 
namics are the Geometric Ornstein-Uhlenbeck (GOU) process and the square root process of Feller (Feller (1951)). The GOU process is governed by

$$
d \ln X_{t}=\lambda\left(\theta-\ln X_{t}\right) d t+\sigma d W_{t}, \quad X_{0}=x_{0}
$$

The GOU has gained popularity because it is a continuous version of the EGARCH process (Nelson (1990)), which is often used as a model of the market variance process.

The process $\left\{\ln X_{t}\right\}_{t \geq 0}$ is an Ornstein-Uhlenbeck process (OU). The transition density functions of the OU process have Gaussian distributions. Hence, the transition density functions of the GOU have log-normal distributions. Detemple and Osakwe (2000) examine finite-lived European and American options on the volatility under the GOU assumption. Because of the log-normal distributions, vanilla option valuation under the GOU assumption is analytically tractable. However, there is one major limitation of using the GOU to describe the VIX dynamics. Due to the log-normal distributions, the implied volatility of VIX options under the GOU assumption is constant across strikes, which we shall see that this assumption is not empirically tenable for VIX options.

The Feller process is governed by

$$
d X_{t}=\lambda\left(\theta-X_{t}\right) d t+\sigma \sqrt{X_{t}} d W_{t}
$$

In the stochastic volatility field, the process is known as the Heston (1993) model. To guarantee that $\left\{X_{t}\right\}_{t \geq 0}$ cannot reach zero requires $2 \lambda \theta \geq \sigma^{2}$. This inequality is called the Feller condition. The Feller process provides one of the earliest stochastic volatility models. This model is important because the process is mean-reverting with a great deal of tractability. Many derivative products are analytically tractable under the Heston stochastic volatility framework. The steady-state distribution has a gamma distribution (Cox, Ingersoll Jr, and Ross (1985) $)^{7}$. Grün-

${ }^{7}$ For brevity, we choose to omit the details of the gamma distribution, inverse gamma distribution, as well as 
bichler and Longstaff (1996) assume that the dynamics of VIX are driven by a Feller process. Not surprisingly, a process of this kind generates very negatively skewed distributions, as the diffusion term is scaled by $\sqrt{X_{t}}$ instead of $X_{t}$. Unfortunately the most relevant stylized fact of the VIX market is a positive skew of the implied volatility, as documented by Wang and Daigler (2011) and Mencía and Sentana (2013).

Other than the GOU and Feller models, we also assume that the VIX dynamics are driven by an Inhomogeneous Geometric Brownian Motion (IGBM). The reason is that it is engineered to yield a closed-form solution for American VIX option valuation, and compare with their European-style counterparts consequently. The IGBM process satisfies

$$
d X_{t}=\lambda\left(\theta-X_{t}\right) d t+\sigma X_{t} d W_{t}, \quad X_{0}=x_{0}
$$

where $\sigma$ is the instantaneous volatility, $\theta$ the equilibrium mean level, $\lambda$ the speed of reversion and $\left\{W_{t}\right\}_{t \geq 0}$ is a standard Wiener process. Since the discrete version of the model is a GARCH $(1,1)$ process (Nelson (1990)), the process is also known as the GARCH diffusion process. Lewis (2000) uses the diffusion process (3) to describe the stochastic volatility. The process is also adopted to describe the value of real options by Bhattacharya (1978) and by Brennan and Schwartz (1980) to study the instantaneous interest rate.

The boundary 0 is an entrance, and the boundary $\infty$ is natural. Put differently, the process will only take positive values. In fact, using Ito's lemma, it can be demonstrated that an analytical solution is given by

$$
X_{t}=e^{-\left(\lambda+\frac{1}{2} \sigma^{2}\right) t+\sigma W_{t}}\left(X_{0}+\lambda \theta \int_{0}^{t} e^{\left(\lambda+\frac{1}{2} \sigma^{2}\right) s-\sigma W_{s}} d s\right)
$$

This positivity feature is appealing in describing many financial assets, such as volatility. The steady-state of the process $\left\{X_{t}\right\}_{t \geq 0}$ follows an inverse gamma distribution (see, e.g., Nelson

the Feller condition in this paper, which are available in a companion paper of $\operatorname{Yan}$ and Zhang $(2018)$. 


\section{(1990)).}

Let $\kappa=\frac{2 \lambda}{\sigma^{2}}$, the steady-state $X_{\infty}$ of the IGBM process $(3)$ has the density function $\frac{1}{\Gamma(\kappa+1)}(\kappa \theta)^{\kappa+1} x^{-\kappa-2} e^{-\frac{\kappa \theta}{x}}$. This leads to the moment calculations of the steady-state:

$$
\mathbb{E} X_{\infty}^{n}= \begin{cases}\frac{\Gamma(\kappa+1-n)}{\Gamma(\kappa+1)} \kappa^{n} \theta^{n}, & n<\kappa+1 \\ \infty, & \text { otherwise }\end{cases}
$$

The IGBM process generates a positively skewed distribution which we shall find is much better suited to modeling VIX than the first two processes.

\section{Empirical Stylized Facts}

We review the history of the VIX and consider evidence on the suitability of each of the three models looking at the time series of both options prices and the VIX.

The CBOE volatility index (VIX) was introduced in 1993. The index, commonly known as the investor fear factor 8 reflects a market consensus view of the implied volatility of S\&P 500 index options over the next 30 days. On March 26, 2004, the Chicago Futures Exchanges (CFE) launched its first-ever traded VIX futures (VX), which are standard futures contracts. VIX options were launched on Feb 24, 2006. The volume of VIX options is growing at a much faster rate than the VIX futures, and the average daily volume of VIX options $(687,181$ contracts) is

\footnotetext{
${ }^{8}$ We are aware of the existence of two versions of VIX, with a pairwise correlation coefficient larger than 0.99. Initially, VIX is based on the implied volatility of S\&P 100 index At-The-Money (ATM) options, determined by inverting the Black and Scholes (1973) formula. On September 23, 2003, the original calculation of VIX was revamped to accommodate more robustness and back-calculated to 1990, following the model-free calculation method of Demeterfi, Derman, and Kamal (1999); Britten-Jones and Neuberger (2000); Jiang and Tian (2005). The principal idea of favoring the new calculation is based on the fact that variance swaps can be replicated by the corresponding option prices of the underlying. The CBOE kept the VIX as the new volatility index while renamed the old one the VXO. A historical development of these two volatility indices is presented in Carr and $\mathrm{Wu}(2006)$.
} 
about three fold larger than the VIX futures (283,342 contracts) in 2014? They are analogous to the standard options written on stocks. In this study, we obtain the historical time series data of VIX from the CBOE website, which range between January 2, 1990 and September 30, 2012 with a total of 5,732 observations ${ }^{10}$ We collect the VIX option data from Market Data Express (MDE).

\subsection{Implied volatility}

Under an equivalent risk-neutral measure, we cannot use the Black-Scholes model (Black and Scholes (1973)) to describe the VIX dynamics. This is because the VIX is mean-reverting, while the underlying in the Black-Scholes framework can reach indefinitely high levels in contrast. In order to get the implied volatility of VIX, we use the futures dynamics:

$$
d F_{t, T}=\sigma F_{t, T} d W_{t}
$$

where $\left\{W_{t}\right\}_{t \geq 0}$ is a standard Brownian Motion process, $\sigma$ is the instantaneous volatility and $T$ is the maturity. SDE (4) is also called the Black model (Black (1976)). The only unknown variable appearing in the Black model is the constant volatility $\sigma$. However, it is widely acknowledged that the volatility is far from constant. This empirical difficulty leads to the development of implied volatility. The implied volatility connects the Black option price with the corresponding market option price. Precisely speaking, the implied volatility is defined to be the value with which the Black option price at a given strike $K$ (or $\log$ moneyness $k:=\ln \frac{K}{F_{0, T}}$ ) and expiration $T$ matches the corresponding market option price. The total implied variance is defined to be the implied variance multiplied by the time to expire.

\footnotetext{
${ }^{9}$ http://markets.businessinsider.com/news/stocks/VIX-Options-and-Futures-Set-New-Daily-VolumeRecords-1002250217.

${ }^{10}$ Our sample period is comparable to the latest literature such as Mencía and Sentana (2013) and Branger, Kraftschik, and Völkert (2016).
} 
To intuitively understand the shortcomings of the existing models such as the GOU and Feller models as well as the improvements provided by our IGBM model, it is instructive to graphically show some of the models' statistical properties that are key for option pricing. As a representative example, Figure 1a plots the total implied variances of VIX options on March 24,2010 . The implied variance curve is upward sloping, suggesting the slope of the implied volatility is positive. The positive slope means that volatility tends to have a heavier right tail distribution. Figure $1 \mathrm{~b}$ reports the total implied variances of VIX options against log moneyness for the three dynamics. The implied variance curve from the Feller process is downward sloping from left to right. The slope of implied volatility is negative, which is in stark contrast with what is empirically observed in the market. If the dynamics are driven by a GOU process, the implied variance is constant, which is also empirically biased. If we assume the VIX dynamics are driven by an IGBM process, the implied variance curve is upward sloping from left to right, and the slope of implied variance is positive. Overall, the implied volatility examination demonstrates that neither the Feller nor the GOU process but the IGBM model is empirically consistent with the upward sloping implied variance in the markets, which is the most relevant stylized fact of the VIX market, as documented by Wang and Daigler (2011); Mencía and Sentana (2013).

\subsection{Time series analysis}

To add robustness to our estimates, we split the data into two non-overlapping subperiods (shown in the left and right columns) and use two different approaches. The top panel (Panel A) in Table 1 shows the results of fitting each of the three processes to its steady-state distribution; the bottom panel (Panel B) in Table 1 shows the results of fitting changes into the discretized diffusion equation. In each case, we use the maximum likelihood method. In the top panel (Panel A) in Table 1, the maximum likelihood method is carried out to estimate steady-state distributions of these processes. Table 2 summarizes the the stationary state distributions. However, the steady-state distributions depend only on $\theta$ and $\kappa$. To estimate the volatility $\sigma$, we apply Ito's Lemma to first transform the original process to the one whose Brownian coefficient is $\sigma$. We then compute the quadratic variation of the transformed process to estimate $\sigma$. Table 2 reports the transformed process and the quadratic variation. The 
bottom panel (Panel B) in Table 1 shows the maximum likelihood method which is directly based on the transition density functions proposed by Aït-Sahalia (2002) and Yu (2007).

Table 1 reports the calibrated parameter values. The Feller condition is satisfied, which means that zero is not attainable for the Feller model. The estimated coefficients are qualitatively similar across different periods and different methods, though there are slightly slower reversions in the second period. More importantly, the IGBM provides a significantly better fit in both sub-periods than the other two models, while the Feller model is the worst fit in both periods. Over the whole sample period, our calibrated parameters from the maximum likelihood method proposed by Aït-Sahalia (2002) and Yu (2007) are $\lambda=3.625, \theta=0.205$, $\sigma=0.965$. We take them to further analysis throughout this paper, although our results are robust to other parameters. ${ }^{11}$

\section{Perpetual American Options}

Perpetual American options are limiting cases of finite-lived American options. They have no expiration date and can be exercised at any time. Assuming the value process of the underlying asset is $\left\{X_{t}\right\}_{t \geq 0}$ and the strike price is $K$, a perpetual American call pays $\left(X_{t}-K\right)^{+}$if it is exercised at time $t$, while a perpetual American put pays $\left(K-X_{t}\right)^{+}$if it is exercised at time t. The valuation of (perpetual) American options written on stocks was originally investigated by Merton (1973) where he assumed that the stock dynamics are governed by a Geometric Brownian Motion. In option pricing, perpetual American options have at least two obvious advantages over conventional American options. First, unlike finite-lived American options, perpetual American options admit tractable solutions. Consequently, they provide an analyti-

\footnotetext{
${ }^{11}$ In Table 1] for the GOU model, the estimated value for $\theta$ is negative. This is the estimated long-run mean for the natural log of volatility, not for the level of volatility. To convert this number to a volatility level and make it comparable to the long-run mean estimates for the Feller and IGBM models, we only need to take the exponential of the negative numbers (i.e., $-1.684,-1.613,-1.678,-1.623$ ) to get rid of the logarithm. The comparable numbers are $0.186,0.199,0.187,0.197$, respectively.
} 
cal view on how the changes of the underlying index, the speed of reversion, the equilibrium mean level and the volatility affect the American option values. Second, they offer asymptotic boundaries for their finite-lived counterparts.

The pricing of perpetual American options relates to the theory of optimal stopping (see, e.g., Peskir and Shiryaev (2006) $)$. The general approach is well-established. There are also some precedents for valuing perpetual American options on mean-reverting processes, particularly in the real options literature (see, e.g., Dixit and Pindyck (1994)). Since the IGBM provides an empirically consistent implied volatility, we use the IGBM process to price perpetual American VIX options.

Let $r$ be the constant risk-adjusted rate of discount, the optimal present value of the perpetual American option is given by

$$
V(x)=\sup _{\tau} \mathbb{E}_{x}\left(e^{-r \tau} f\left(X_{\tau}\right)\right)
$$

where the supremum is taken over stopping time $\tau$ who satisfies $\tau \geq 0, f(\cdot)$ is the payoff when the option is exercised and $x$ represents the initial level of the process. This optimal stopping problem is equivalent to finding a barrier $h$, such that $V(x)$ achieves the supremum when the process first hits the optimal exercising region. In the case of put options, the stopping time is $\tau_{h}=\inf \left\{t \geq 0: X_{t} \leq h\right\}$. In the case of call options, the stopping time is $\tau_{h}=\inf \left\{t \geq 0: X_{t} \geq\right.$ $h\}$. The unique value $h$ is the critical value, the threshold value or the optimal exercise boundary. Replacing the stopping time $\tau_{h}$ to Equation (5), we have

$$
V(x)=\mathbb{E}_{x}\left(e^{-r \tau_{h}} f\left(X_{\tau_{h}}\right)\right)=\mathbb{E}_{x}\left(e^{-r \tau_{h}}\right) f(h) .
$$

In the region where it is not yet optimal to exercise, the value function satisfies

$$
\lambda(\theta-x) \frac{d}{d x} V(x)+\frac{1}{2} \sigma^{2} x^{2} \frac{d^{2} V}{d x^{2}}=r V(x)
$$


12

By Lemma 1, the solution to (6) takes the form

$$
V(x)=A x^{-a} M\left(a, b ; \frac{c}{x}\right)+B x^{-a} U\left(a, b ; \frac{c}{x}\right) .
$$

where $M(a, b ; x)$ and $U(a, b ; x)$ are confluent hypergeometric functions of the first and second kinds respectively (see Appendix B) and

$$
a=\frac{\sqrt{\left(2 \lambda+\sigma^{2}\right)^{2}+8 r \sigma^{2}}-\left(2 \lambda+\sigma^{2}\right)}{2 \sigma^{2}}, \quad b=\frac{2 \lambda}{\sigma^{2}}+2 a+2, \quad c=\frac{2 \lambda \theta}{\sigma^{2}} .
$$

In order to determine $A$ and $B$, we shall need the boundary conditions. The two cases of put and call options are treated separately in the following two subsections.

\footnotetext{
${ }^{12}$ Under a general time-homogeneous diffusion

$$
d X_{t}=\mu\left(X_{t}\right) d t+\sigma\left(X_{t}\right) d W_{t}
$$
}

the discounted expectation of a contingent payoff $V\left(X_{T}, T\right)$ under a constant interest rate $r$ is a function $V\left(X_{t}, t\right)$ which evolves (backwards in time) under the Feyman-Kac equation

$$
\frac{\partial V(x, t)}{\partial t}+\mu(x) \frac{\partial}{\partial x} V(x, t)+\frac{1}{2} \sigma^{2}(x) \frac{\partial^{2} V(x, t)}{\partial x^{2}}-r V(x, t)=0
$$

The Black-Scholes partial differential equation is a special case of this. This equation also applies to the evolution of American option values in the region where it is not yet optimal to exercise. For perpetual options, its value no longer depends on time, because it is always an infinite time away from expiration. We can write its value at any time as $V(x)$ instead of $V(x, t)$. For our IGBM process, this specializes to:

$$
\lambda(\theta-x) \frac{d}{d x} V(x)+\frac{1}{2} \sigma^{2} x^{2} \frac{d^{2} V}{d x^{2}}-r V(x)=0 .
$$

This equation holds in the region where it is not optimal to exercise. There is free boundary problem of where it is optimal to exercise subject to other boundary conditions which characterize the value when it is exercised. 


\subsection{Perpetual American put options}

Let us change the previous $V(x)$ to $P(x)$ to make it clear that this is the value for perpetual put options. The boundary conditions are:

$$
\begin{gathered}
P(h)=K-h, \\
P^{\prime}(h)=-1, \\
P(x) \rightarrow 0, \text { as } x \rightarrow \infty
\end{gathered}
$$

Equation (8) is called the value matching condition. Equation (9) is called the smooth pasting condition, which states that the optimal barrier is selected such that the value function $V(x)$ is smooth around that point. In other words, the slope of the value function exactly equals the slope of the exercise value (Merton (1973)). Condition (10) holds, because $\infty$ is the natural boundary. A natural boundary is a point in which the process can neither reach in finite mean time nor can it be started from (Karlin and Taylor (1981)). Thus, if the process starts from the natural boundary, it will take an infinite mean time to reach any interior point. Since $b>2$, the boundary condition (10) and the asymptotic expansion (B.1) imply $B=0$.

We show that the solution $h$ that satisfies the conditions (8) and (9) is unique. The recursive relationship $(v-u) M(u, v+1 ; x)=v M(u, v ; x)-v M^{\prime}(u, v ; x)$ and $\frac{P(h)}{P^{\prime}(h)}=h-K$ yield

$$
\frac{c(b-a)}{b} \frac{M\left(a, b+1 ; \frac{c}{h}\right)}{M\left(a, b ; \frac{c}{h}\right)}=\frac{-(a+1) h^{2}+(a K-c) h+c K}{K-h} .
$$

Proposition 1. The solution $h$ to Equation 11 exists and is unique. Moreover, we have $K \frac{\sqrt{a+1}-1}{\sqrt{a+1}}<$ $h<K$.

The condition (8) implies

$$
A=\frac{K-h}{h^{-a} M\left(a, b ; \frac{c}{h}\right)}
$$


therefore, we get the optimal present value for the perpetual put option

$$
P(x)= \begin{cases}(K-h) \frac{x^{-a} M\left(a, b ; \frac{c}{x}\right)}{h^{-a} M\left(a, b ; \frac{c}{h}\right)}, & \text { if } x>h ; \\ K-x, & \text { if } 0<x \leq h .\end{cases}
$$

Note that to calculate the solution for a given strike, we first find the value of $h$ which solves equation (11). To find $h$, we can use some numerical methods, such as the bisection algorithm, to search within the bounded range $\left(K \frac{\sqrt{a+1}-1}{\sqrt{a+1}}, K\right)$.

\subsection{Perpetual American Call Options}

For the same reason, we change the previous $V(x)$ to $C(x)$ to make it clear that this is the value of call options. The boundary conditions are:

$$
\begin{gathered}
C(h)=h-K, \\
C^{\prime}(h)=1, \\
C(x)<\infty \text {, as } x \rightarrow 0+.
\end{gathered}
$$

Again, Equation (14) comes from the smooth fit principle, and condition (15) holds since 0 is the entrance. An entrance boundary is a point if the process begins there, it quickly moves to the interior of the state space and never returns to that point any more (Karlin and Taylor (1981)). The asymptotic behavior (B.3) and the boundary condition (15) imply $A=0$. We show the solution $h$ that satisfies the conditions (13) and (14) is unique. The derivative $U^{\prime}(u, v ; x)=$ $-u U(u+1, v+1 ; x)$ and $\frac{C(h)}{C^{\prime}(h)}=h-K$ yield

$$
\frac{U\left(a+1, b+1 ; \frac{c}{h}\right)}{U\left(a, b ; \frac{c}{h}\right)}=\frac{(a+1) h^{2}-a K h}{a c(h-K)} .
$$

Proposition 2. The solution $h$ to Equation (16) exists and is unique. Furthermore, $h>\frac{b-a-1}{b-a-2} K$. 
The condition (13) implies

$$
B=\frac{h-K}{h^{-a} U\left(a, b ; \frac{c}{h}\right)},
$$

which implies the optimal present value of the perpetual American call option has the expression given by

$$
C(x)= \begin{cases}(h-K) \frac{x^{-a} U\left(a, b ; \frac{c}{x}\right)}{h^{-a} U\left(a, b ; \frac{c}{h}\right)}, & \text { if } x<h ; \\ x-K, & \text { if } x \geq h .\end{cases}
$$

To value the calls, we also have an easy bisection search ${ }^{13}$ for the value of $h$ which solves equation (16) following by evaluating equation (17).

\subsection{Sensitivity analysis of perpetual American options}

We adopt the standard Greeks in finance to analyze the sensitivity of the value function $V(x)$ to changes in different parameters. The option delta, $\frac{\partial V(X)}{\partial x}$, measures the rate of change of the value function with respect to a change in the underlying asset price. The option gamma, $\frac{\partial^{2} V(x)}{\partial x^{2}}$, is the rate of change of the delta with respect to a change in the underlying asset price. Since it is easy to differentiate both the $M(a, b ; x)$ and $U(a, b ; x)$ functions with respect to their final arguments, we can give the following expressions for the delta and gamma of each option.

\section{Proposition 3.}

$$
\begin{gathered}
\frac{\partial P(x)}{\partial x}= \begin{cases}-\frac{a}{x} \frac{M\left(a+1, b ; \frac{c}{x}\right)}{M\left(a, b ; \frac{c}{x}\right)} P(x), & \text { if } x>h ; \\
-1, & \text { if } 0<x \leq h .\end{cases} \\
\frac{\partial^{2} P(x)}{\partial x^{2}}= \begin{cases}\frac{a(a+1)}{x^{2}} \frac{M\left(a+2, b ; \frac{c}{x}\right)}{M\left(a, b ; \frac{c}{x}\right)} P(x), & \text { if } x>h ; \\
0, & \text { if } 0<x \leq h .\end{cases}
\end{gathered}
$$

\footnotetext{
${ }^{13}$ Note the value $\frac{1}{h}$ is bounded between 0 and $\frac{b-a-2}{b-a-1} \frac{1}{K}$.
} 


\section{Proposition 4.}

$$
\begin{aligned}
& \frac{\partial C(x)}{\partial x}= \begin{cases}\frac{a(b-a-1)}{x} \frac{U\left(a+1, b ; \frac{c}{x}\right)}{U\left(a, b ; \frac{c}{x}\right)} C(x), & \text { if } 0<x<h ; \\
1, & \text { if } x \geq h .\end{cases} \\
& \frac{\partial^{2} C(x)}{\partial x^{2}}= \begin{cases}\frac{a(a+1)(b-a-1)(b-a-2)}{x^{2}} \frac{U\left(a+2, b ; \frac{c}{x}\right)}{U\left(a, b ; \frac{c}{x}\right)} C(x), & \text { if } 0<x<h ; \\
0, & \text { if } x \geq h .\end{cases}
\end{aligned}
$$

They have the expected properties and are easy to evaluate. The partial derivatives with respect to $\sigma, \theta$ and $\lambda$ are mathematically more complicated. To get these sensitivities, we examine the asymptotic value of the optimal exercising boundary when the relevant parameter approaches either 0 or $\infty$.

Proposition 5. Let $h$ be the critical value of perpetual American put option, then

$$
\begin{array}{lll}
\lim _{\lambda \rightarrow 0+} h=\frac{\sqrt{\sigma^{2}+8 r}-\sigma}{\sqrt{\sigma^{2}+8 r}+\sigma} K, & \lim _{\sigma \rightarrow 0+} h=\min \left\{K, \frac{\lambda \theta+r K}{r+\lambda}\right\}, & \lim _{\theta \rightarrow 0+} h=\frac{a K}{a+1}, \\
\lim _{\lambda \rightarrow+\infty} h=\min \{K, \theta\}, & \lim _{\sigma \rightarrow+\infty} h=0, & \lim _{\theta \rightarrow+\infty} h=K .
\end{array}
$$

Let $h$ be the critical value of perpetual American call option, then

$$
\begin{aligned}
& \lim _{\lambda \rightarrow 0+} h=\frac{\sqrt{\sigma^{2}+8 r}+\sigma}{\sqrt{\sigma^{2}+8 r}-\sigma} K, \quad \lim _{\sigma \rightarrow 0+} h=\max \left\{K, \frac{\lambda \theta+r K}{r+\lambda}\right\}, \quad \lim _{\theta \rightarrow 0+} h=\frac{b-a-1}{b-a-2} K, \\
& \lim _{\lambda \rightarrow+\infty} h=\max \{K, \theta\}, \quad \lim _{\sigma \rightarrow+\infty} h=\infty, \quad \lim _{\theta \rightarrow+\infty} h=\infty \text {. }
\end{aligned}
$$

Proposition 5 offers an intuitive view of the behaviors of critical value to a change of these parameters. The impact of the volatility on the option pricing is easy to understand. When volatility increases, the process is more likely to move into a broader region for a given period of time. Thus, increasing the volatility $\sigma$ increases the value of both kinds of options.

The equilibrium level and the speed of reversion are unique for a mean-reverting asset. It is worth discussing their roles. As expected, decreasing the equilibrium level of the process increases the value of the puts and decreases that of the calls. 
The speed of reversion measures the strength of the restoring force by which it pulls the process toward the equilibrium mean level. A higher level of the speed of reversion means the force is stronger and the process will be more likely to move within a narrow scope of the equilibrium mean level. Any departure from the equilibrium mean level becomes difficult. In contrast, a lower level of the speed of reversion means that the restoring force is weaker. Due to the Brownian Motion shock, the process is likely to move in a wide range around the equilibrium mean. When the initial level is far away from the equilibrium mean level, it may take a considerably longer time for the process with a lower speed of reversion to move back to the equilibrium mean than it would a process with a higher speed of reversion. The tradeoff between moving into a wider range and taking a longer period suggests that the option prices, with respect to the speed of reversion, might be mixed. Indeed, depending on the chosen parameters, as a function of the speed of reversion, Proposition 5 shows that the critical value can be either monotonically increasing or monotonically decreasing. In addition, it can be initially increasing and eventually decreasing in the case of call options, and initially decreasing and eventually increasing in the case of put options. These different possibilities lead to mixed results when considering the relation between the speed of reversion and the perpetual options.

Figure 2 shows the sensitivity of the put and call curves to each of the three process parameters. Within a reasonable range of parameters, a lower speed of reversion is associated with a lower (higher) critical value and a higher perpetual option value for put (call) options.

\subsection{Mean-first passage time}

In pricing perpetual options, it is interesting to know the length of the exercise time. A measurement of the optimal exercise time is the Mean First-Passage Time (MFPT). Knowing MFPT will allow investors to know the expected time the option is exercised. In order to get the MFPT, we compute the Laplace transform of the First-Passage Time (FPT). The relation between the Laplace transform of FPT and the MFPT is established by equation

$$
\mathbb{E}_{x}\left(\tau_{y}\right)=-\lim _{r \rightarrow 0+} \frac{\partial \mathbb{E}_{x}\left(e^{-r \tau_{y}}\right)}{\partial r}
$$


The Laplace transform $\mathbb{E}_{x}\left(e^{-r \tau_{y}}\right)$ is essentially the discounted present value at the time the option is exercised. The MFPT is the marginal rate change of the discounted present value as the riskless interest rate approaches zero.

From the perpetual put and perpetual call values, the discounted present value is given by

$$
\mathbb{E}_{x}\left(e^{-r \tau_{h}}\right)= \begin{cases}\frac{P(x)}{K-h}=\frac{x^{-a} M\left(a, b ; \frac{c}{x}\right)}{h^{-a} M\left(a, b ; \frac{c}{h}\right)}, & x \geq h ; \\ \frac{C(x)}{h-K}=\frac{x^{-a} U\left(a, b ; \frac{c}{c}\right)}{h^{-a} U\left(a, b ; \frac{c}{h}\right)}, & x<h .\end{cases}
$$

Using a reasonable set of parameter values, the critical value, the MFPT and the perpetual option values for several strikes and initial levels under the IGBM assumption are reported in Table 3. It seems that the average time to optimally exercise perpetual options on volatility is quite long. This stems from the process followed by volatility. In the short-term, high levels of volatility tend to be followed by high ones, low levels of volatility tend to be followed by low ones and volatility does not depart too far away from the starting level. In the long-term, however, the mean-reverting property dominates. The volatility in the long-term can achieve both high and low values. Thus, for perpetual American options, the optimal time to exercise is on average quite long.

\section{Finite-lived American Options}

This section valuates American options with finite expiries. Unlike perpetual options, no analytical solutions to finite-lived American options are available. Either numerical or approximation methods are generally taken. Kim (1990), Carr, Jarrow, and Myneni (1992) decompose an American option into the corresponding European option and the early exercise premium. They then use the numerical method to solve the early exercise premium recursively. Alternatively, Barone-Adesi and Whaley (1987) derive the result of the early exercise premium in the perpetual case after approximating the exercise premium by a quadratic function.

More straightforward, we use a binomial lattice method (Cox, Ross, and Rubinstein (1979)) to price American options directly. Nelson and Ramaswamy (1990) propose a binomial lattice 
method for a general diffusion process. A computationally simple binomial tree can be constructed when the volatility of the underlying process $\left\{X_{t}\right\}$ is constant. When the volatility of the underlying process $\left\{X_{t}\right\}$ is level dependent, the total displacement from an initial downward movement followed by an upward movement does not reconnect from an initial upward movement followed by a downward movement. As a consequence, the number of binomial nodes doubles at each time step. It is costly in computation. To overcome the computational complexity, Nelson and Ramaswamy (1990) transform the original process $\left\{X_{t}\right\}$ to a new one in which the volatility component is constant. Once the displacements of an upward movement and a downward movement are specified, the upward movement and down movement probabilities are specified to match the drift of the transformed process. The transformed process is converted back to the original one to value options.

There are also some technical adjustments when the volatility of the original process has singularity points. The singularity is a boundary point of the spot price at which the volatility term vanishes or explodes. Without the adjustment, the upward movement displacement of the original process from a binomial node close to the low boundary point is so small that it is hard to match the drift term. In other words, the upward movement probability will not lie between 0 and 1. To adjust it, Nelson and Ramaswamy (1990) assume several upward movements to match the drift and to ensure that the upward movement probability is valid when the spot is close to the low boundary. A similar procedure is adopted for the downward movement when the spot is close to the upward boundary. Following their methodology, we convert the original process to the one we report in Table 2. We use the converted process to build a binomial tree ${ }^{14}$ and price finite-lived American options.

Figure 3 plots the values of American call and put options against the initial value of the underlying for different times to expire. Naturally, as these are American options, the perpetual ones have the highest value, providing an upper bound for the finite expiration ones which nest underneath. In order to obtain reasonable vertical spacings on the two figures we have used a

\footnotetext{
${ }^{14}$ In Table 2 the Brownian coefficient of the converted process is $\sigma$, but 1 in Nelson and Ramaswamy (1990).
} 
coarser spacing of expiration periods for the calls than the puts. The length of the expiration period makes a large difference in the pricing the call options, even for very low strikes.

For conventional expiration periods, the put options lie much closer to the perpetual case than do the calls. This comes about because of the positively skewed nature of our distributions. The behavior of the asymptotes (puts at high strikes and calls at low ones) is also interesting. Because of the mean-reverting nature of the process, it is rarely optimal to exercise immediately.

\section{Alternative Mean-Reverting Processes}

Throughout the paper, we assume that the dynamics of VIX are driven by an IGBM process. The natural question of model risk/uncertainty arises. How different are our results if the dynamics of VIX are governed by a different mean-reverting process?

Alternatively, we consider that the dynamics of VIX are governed by the Feller process and the GOU process. Figure 4 compares both European and American options across the three different assumptions for the dynamics. The figure shows that, other things being equal, American options are more expensive than their European counterparts. The excess value reflects an early exercise premium. Based on the implied volatility study in Section 11, the implied volatility is upward sloping for the IGBM process, while it is downward sloping for the Feller process and constant for the GOU process. Translating into the distribution of VIX, the IGBM process places more density weights on the high level than the Feller process and the GOU process; the Feller process places more weights on the low level than the IGBM process and the GOU process.

For the European options, as we expected, these results in the IGBM model giving higher values than other models for out-of-the-money (high strike) call options, and lower ones for out-of-the-money (low strike) put options. The American options exhibit a greater divergence across the three models illustrating the importance of the nature of the paths as well as the distribution of the underlying at the expiration date.

The valuation of American options under mean-reverting processes also depends on the 
nature of the boundaries of the dynamic process. Since the call option is monotonically increasing at the underlying price, the minimum call option is achieved when the starting level is 0 . If the point 0 is a natural boundary, the minimum call option value is 0 . However, if the point 0 is an entrance, the minimum call option value is strictly positive. Similarly, as the put option is monotonically decreasing at the underlying price, the minimum put value is realized when the underlying price approaches $\infty$. If the point $\infty$ is an entrance, the put option always has a strictly positive minimum value. However, if the point $\infty$ is a natural boundary, the minimum put option is 0 . Table 4 lists the boundary types for the three mean-reverting processes.

\section{Concluding Remarks}

This study explores the pricing of American VIX options by investigating the roles of the equilibrium level, the speed of reversion, and the volatility and expiration date in the valuation. It is essential to have a simple yet robust model that is relevant from both a theoretical and practical perspective, and we believe that our IGBM model satisfies this criterion. At least it is able to capture the positively skewed Black-Scholes implied volatility of VIX options, while neither the Heston process nor the process with log-normal distributions is able to capture this empirical stylized fact.

We provide closed-form solutions for perpetual American options. The impact of the equilibrium mean level on the pricing of American VIX options is straightforward. Increasing the

\footnotetext{
${ }^{15}$ Similarly, Mencía and Sentana (2013) conclude that "We initially focus on the two existing mean-reversion models: the square root (SQR) and the log-normal Ornstein-Uhlenbeck processes(LOU). Although SQR is more popular in the empirical literature, we find that the LOU model yields a better fit, especially during the crisis. However, both models yield large price distortions during the crisis. In addition, they do not seem to capture either the level or the slope of the term structure of futures prices, or indeed the volatility skews. ... In this sense, we show that the simple $A R(1)$ structure that they imply in discrete time is not consistent with the empirical evidence of ARMA dynamics for the VIX. In addition, these models are also inconsistent with the strong presence of GARCH-type heteroskedasticity that we find in this volatility index."
} 
equilibrium mean level will increase both the critical values of the call and the put, which results in the drop of put option value and the rise of call option value. The influence of the speed of reversion on the pricing of American VIX options is mixed. However, using a reasonable range of parameters, our calibrations show that increasing the speed of reversion will both drop the call and put option values of VIX.

We compute the MFPT of the optimal exercising time of perpetual American options. We find that the MFPT in exercising is quite long. We also investigate two other mean-reverting processes with different properties and examine the pricing of finite-lived American and European options. We find that American option values are more sensitive to the choice of underlying process than their European-style counterparts, which underscores the importance of our proposed IGBM for American VIX options.

While this study contributes to the pricing of options (including real options) with a meanreverting underlying, several limitations have yet to be overcome. For instance, it would be interesting (especially from the perspective of building trading strategies) to extend our one-factor models by adding volatility jump components (see, e.g., Wu (2011)), volatility on volatility (see, e.g., Branger, Kraftschik, and Völkert (2016)), and more factors (see, e.g., Christoffersen, Heston, and Jacobs (2009)), although many researchers (including us) hold the opinion that they may only provide second-order improvements for pricing VIX derivatives. ${ }^{16}$ We follow the mainstream literature such as Mencía and Sentana (2013) to estimate our onefactor models by maximum likelihood, while a closed-form expression for the likelihood may not be available for many of these extensions. We leave them as directions for future research.

\footnotetext{
${ }^{16}$ For instance, Mencía and Sentana (2013) conclude that "We also find evidence of time-varying volatility in the VIX. As expected, stochastic volatility plays a much more important role for options while leaving futures prices almost unaffected. The CSQR model, though, seems unable to generate the positive slope of the option-implied volatility skews. It is also worth mentioning that jumps only provide a minor improvement for options and do not change futures prices (up to first order). Nevertheless, we would like to emphasise that jumps seem to be a relevant feature to describe the historical dynamics of the VIX, even though they only yield second-order gains for pricing VIX derivatives."
} 


\section{Reference}

Abramowitz, M., and I. A. Stegun. 1972. Handbook of mathematical functions with formulas, graphs, and mathematical tables. Dover, New York.

Aït-Sahalia, Y. 2002. Maximum likelihood estimation of discretely sampled diffusions: A closedform approximation approach. Econometrica 70:223-62.

Aït-Sahalia, Y., and R. Kimmel. 2007. Maximum likelihood estimation of stochastic volatility models. Journal of Financial Economics 2:413-52.

Andersen, T., L. Benzoni, and J. Lund. 2002. An empirical investigation of continuous-time equity return models. Journal of Finance 57:1239-84.

Bakshi, G., C. Cao, and Z. Chen. 1997. Empirical performance of alternative option pricing models. Journal of Finance 52:2003-49.

Barone-Adesi, G., and R. E. Whaley. 1987. Efficient analytic approximation of american option values. Journal of Finance 42:301-20.

Bates, D. S. 2000. Post-'87 crash fears in the s\&p 500 futures option market. Journal of Econometrics 94:181-238.

Bates, R. 1996. Jumps and stochastic volatility: Exchange rate processes implicit in deutsche mark options. Review of Financial Studies 9:69-107.

Bhattacharya, S. 1978. Project valuation with mean-reverting cash flow streams. Journal of Finance 33:1317-31.

Black, F. 1976. The pricing of commodity contracts. Journal of Financial Economics 3:167-79.

Black, F., and M. S. Scholes. 1973. The pricing of options and corporate liabilities. Journal of Political Economy 81:637-54.

Branger, N., A. Kraftschik, and C. Völkert. 2016. The fine structure of variance: Pricing vix derivatives in consistent and log-vix models. SSRN working paper Available at SSRN: https://ssrn.com/abstract $=2028285$.

Brennan, M. J., and E. S. Schwartz. 1980. Analyzing convertible bonds. Journal of Financial and Quantitative Analysis 15:907-29.

Britten-Jones, M., and A. Neuberger. 2000. Option prices, implied price processes, and stochastic volatility. Journal of Finance 55:839-66.

Broadie, M., M. Chernov, and M. Johannes. 2007. Model specification and risk premia: Evidence from futures options. Journal of Finance 62:1453-90. 
Carr, P., R. Jarrow, and R. Myneni. 1992. Alternative characterizations of american put options. Mathematical Finance 2:87-106.

Carr, P., and D. Madan. 1998. Towards a theory of volatility trading. In R. Jarrow, ed., Volatility: New Estimation Techniques for Pricing Derivatives, Risk Publications, 29, 417-27.

Carr, P., and L. Wu. 2004. Time-changed levy processes and option pricing. Journal of Financial Economics 71:113-41.

- 2006. A tale of two indices. Journal of Derivatives 13:13-29.

Chernov, M., A. R. Gallant, E. Ghysels, and G. Tauchen. 2003. Alternative models for stock price dynamics. Journal of Econometrics 116:225-57.

Christoffersen, P., S. Heston, and K. Jacobs. 2009. The shape and term structure of the index option smirk: Why multifactor stochastic volatility models work so well. Management Science 55:1914-32.

Christoffersen, P., K. Jacobs, C. Ornthanalai, and Y. Wang. 2008. Option valuation with long-run and short-run volatility components. Journal of Financial Economics 90:272-97.

Cox, J. C., J. E. Ingersoll Jr, and S. A. Ross. 1985. An intertemporal general equilibrium model of asset prices. Econometrica 53:363-84.

Cox, J. C., S. A. Ross, and M. Rubinstein. 1979. Option pricing: A simplified approach. Journal of Financial Economics 7:229-63.

Demeterfi, K., E. Derman, and M. Kamal. 1999. A guide to volatility and variance swaps. Journal of Derivatives 6:9-32.

Demeterfi, K., E. Derman, M. Kamal, and J. Zou. 1999. More than you ever wanted to know about volatility swaps. Goldman Sachs Quantitative Strategies Research Notes 41.

Detemple, J., and Y. Kitapbayev. 2018. On american vix options under the generalized $3 / 2$ and $1 / 2$ models. Mathematical Finance forthcoming, available at https://arxiv.org/abs/1606.00530.

Detemple, J., and C. Osakwe. 2000. The valuation of volatility options. Review of Finance $4: 21-50$.

Dixit, A., and R. Pindyck. 1994. Investment under uncertainty. Princeton University Press.

Egloff, D., M. Leippold, and L. Wu. 2010. The term structure of variance swap rates and optimal variance swap investments. Journal of Financial and Quantitative Analysis 45:1279-310.

Eraker, B. 2004. Do stock prices and volatility jump? reconciling evidence from spot and option prices. Journal of Finance 59:1367-404. 
Eraker, B., M. Johannes, and N. Polson. 2003. The impact of jumps in volatility and returns. Journal of Finance 58:1269-300.

Feller, W. 1951. Two singular diffusion problems. Annals of Mathematics 54:173-82.

Grünbichler, A., and F. A. Longstaff. 1996. Valuing futures and options on volatility. Journal of Banking and Finance 20:985-1001.

Heston, S. L. 1993. A closed-form solution for options with stochastic volatility with applications to bond and currency options. Review of Financial Studies 6:327-43.

Huang, J., and L. Wu. 2004. Specification analysis of option pricing models based on timechanged lévy processes. Journal of Finance 59:1405-40.

Jiang, G. J., and Y. S. Tian. 2005. The model-free implied volatility and its information content. Review of Financial Studies 18:1305-42.

Karlin, S., and H. M. Taylor. 1981. A second course in stochastic processes. Gulf Professional Publishing.

Kim, I. J. 1990. The analytic valuation of american options. Review of Financial Studies 3:547-72.

Lewis, A. L. 2000. Option valuation under stochastic volatility. Finance Press, Newport Beach, CA.

Lin, Y.-N., and C.-H. Chang. 2009. Vix option pricing. Journal of Futures Markets 29:523-43.

López, J. L., and P. J. Pagola. 2010. The confluent hypergeometric functions $\mathrm{m}$ (a, b; z) and u (a, b; z) for large b and z. Journal of Computational and Applied Mathematics 233:1570-6.

Mauer, D. C., and S. H. Ott. 1995. Investment under uncertainty: The case of replacement investment decisions. Journal of Financial and Quantitative Analysis 30:581-605.

Mencía, J., and E. Sentana. 2013. Valuation of vix derivatives. Journal of Financial Economics 108:367-91.

Merton, R. C. 1973. An intertemporal capital asset pricing model. Econometrica 41:867-87.

Metcalf, G. E., and K. A. Hassett. 1995. Investment under alternative return assumptions comparing random walks and mean reversion. Journal of Economic Dynamics and Control 19:1471-88.

Nelson, D. B. 1990. Arch models as diffusion approximations. Journal of Econometrics 45:7-38.

Nelson, D. B., and K. Ramaswamy. 1990. Simple binomial processes as diffusion approximations in financial models. Review of Financial Studies 3:393-430. 
Neuberger, A. 1994. The log contract. Journal of Portfolio Management 20:74-80.

Neuberger, A. J. 1990. Volatility trading. London Business School working paper.

Pagan, A. R., and G. W. Schwert. 1990. Alternative models for conditional stock volatility. Journal of Econometrics 45:267-90.

Pan, J. 2002. The jump-risk premia implicit in options: evidence from an integrated time-series study. Journal of Financial Economics 63:3-50.

Peskir, G., and A. Shiryaev. 2006. Optimal stopping and free-boundary problems. Springer.

Santa-Clara, P., and S. Yan. 2010. Crashes, volatility, and the equity premium: Lessons from s\&p 500 options. Review of Economics and Statistics 92:435-51.

Sarkar, S. 2003. The effect of mean reversion on investment under uncertainty. Journal of Economic Dynamics and Control 28:377-96.

Schwert, G. W. 1990. Stock returns and real activity: A century of evidence. Journal of Finance 45:1237-57.

-2011. Stock volatility during the recent financial crisis. European Financial Management 17:789-805.

Wang, Z., and R. T. Daigler. 2011. The performance of vix option pricing models: empirical evidence beyond simulation. Journal of Futures Markets 31:251-81.

Whaley, R. E. 1993. Derivatives on market volatility: Hedging tools long overdue. Journal of Derivatives 1:71-84.

Wu, L. 2011. Variance dynamics: Joint evidence from options and high-frequency returns. Journal of Econometrics 160:280-7.

Yan, C., and H. Zhang. 2018. Modeling fundamental analysis into portfolio selection. Quantitative Finance forthcoming, available at http://dx.doi.org/10.2139/ssrn.2755636.

Yu, J. 2007. Closed-form likelihood approximation and estimation of jump-diffusions with an application to the realignment risk of the chinese yuan. Journal of Econometrics $141: 1245-80$.

Zhu, S.-P., and G.-H. Lian. 2012. An analytical formula for vix futures and its applications. Journal of Futures Markets 32:166-90. 


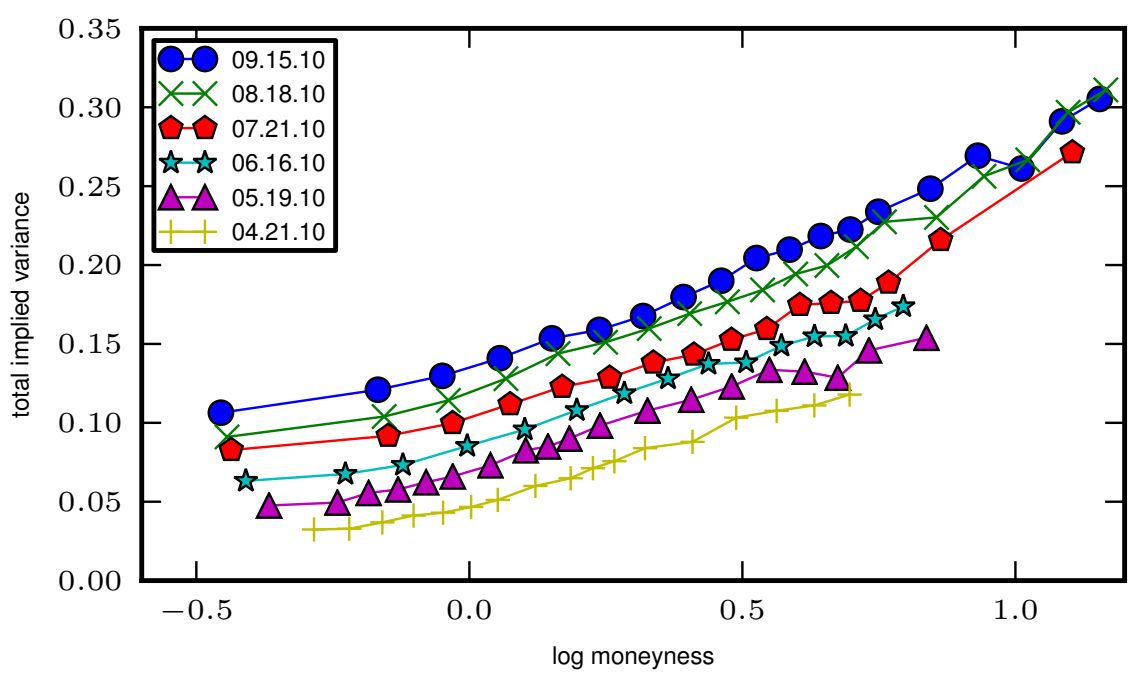

(a) Total implied variances of VIX on March 24, 2010

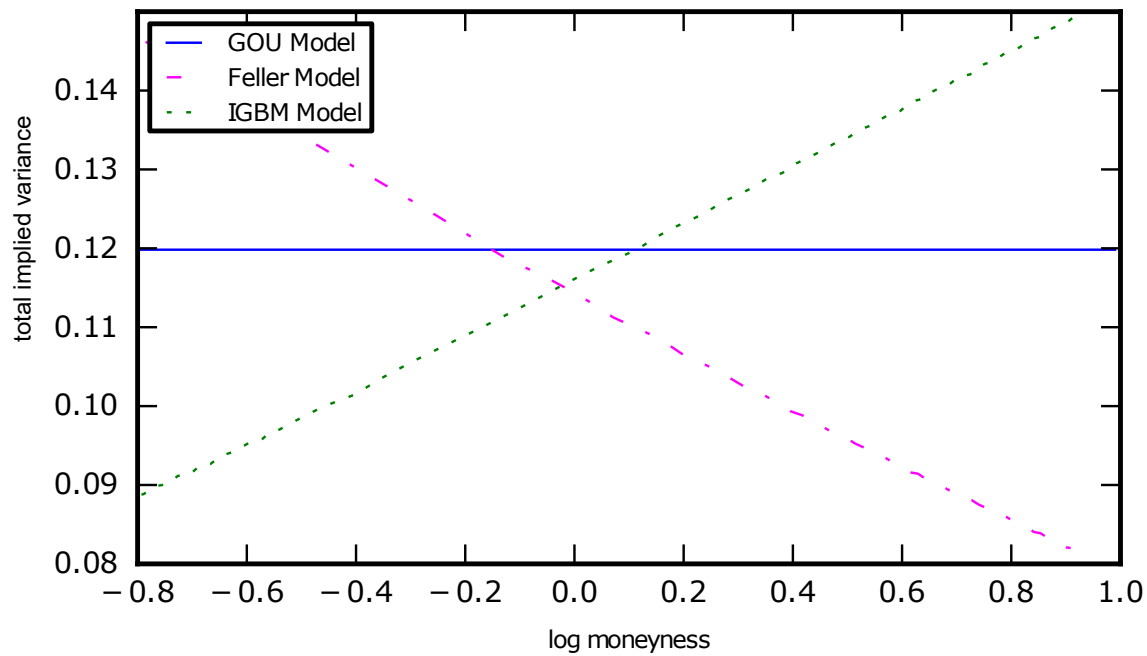

(b) Total implied variances of European VIX options against log moneyness

Figure 1

Total implied variances of VIX options against log moneyness for the three processes.

Figure 1a plots the total implied variances of VIX options on March 24, 2010, while Figure 1b reports the total implied variances of VIX options against $\log$ moneyness (i.e., $k:=\ln \frac{K}{F_{0, T}}$ ) for the three mean-reverting processes. The dates in the legend in the upper left corner of Figure $1 \mathrm{~b}$ correspond to the expiration dates of the option contracts. In Figure $1 \mathrm{~b}$, the base parameters are $T=0.5, r=6 \%, X_{0}=0.2$. For the GOU process, $\lambda=3.832, \theta=$ $-1.651, \sigma=0.969$. For the Feller process, $\lambda=4.605, \theta=0.205, \sigma=0.469$. For the IGBM process, $\lambda=3.625$, $\theta=0.205, \sigma=0.965$. $\sigma$ is the instantaneous volatility, $\theta$ the equilibrium mean level, and $\lambda$ the speed of reversion. 
Sensitivity to the speed of reversion
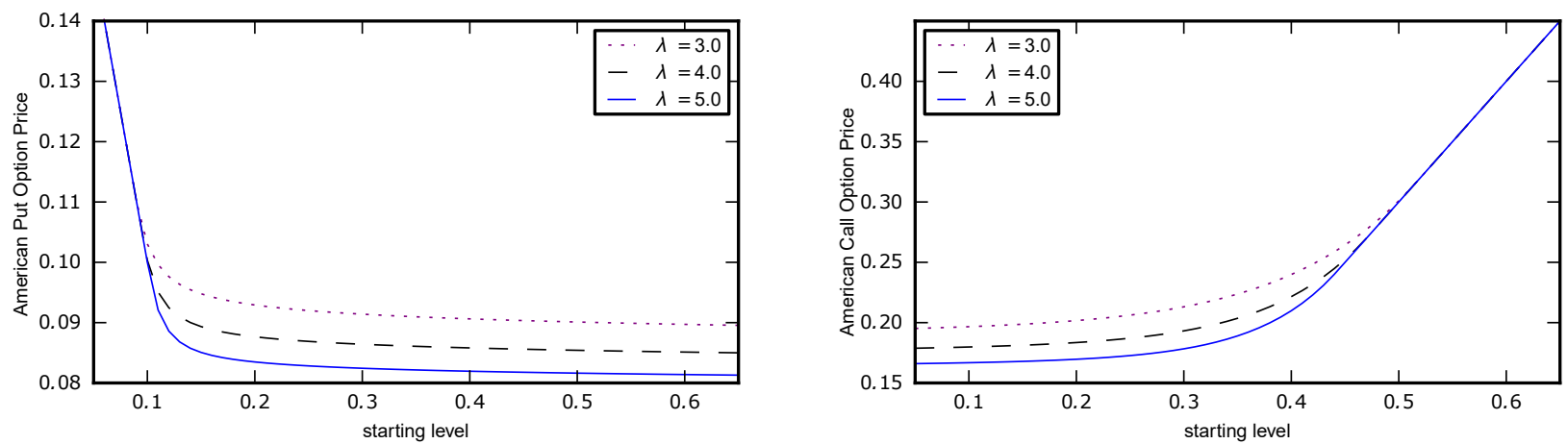

Sensitivity to the equilibrium mean
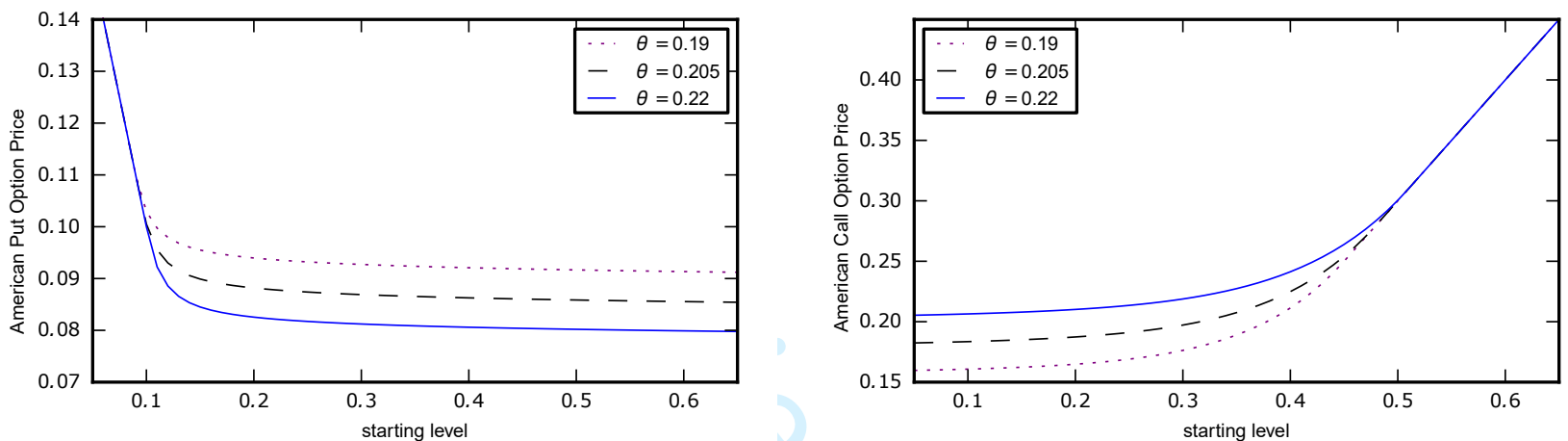

Sensitivity to the volatility

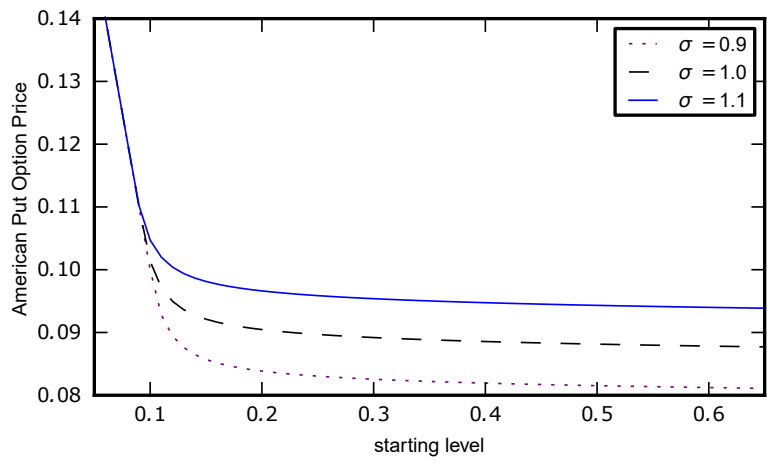

Perpetual American put options

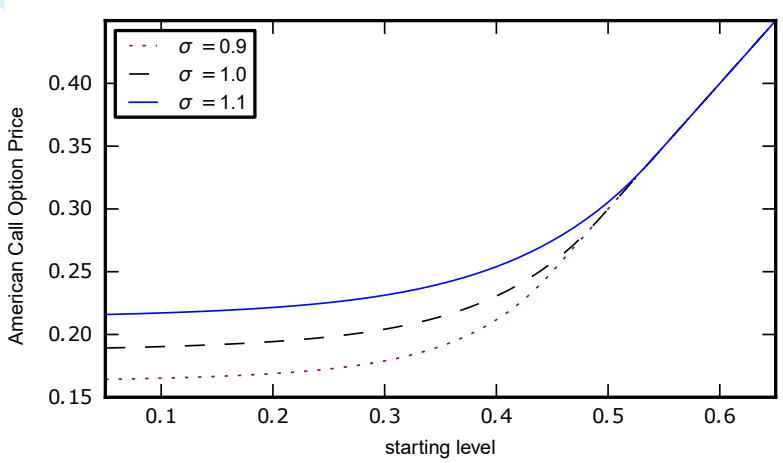

Perpetual American call options

Figure 2

\section{Sensitivity analysis of perpetual American options for the IGBM process.}

The base parameters are: $K=20 \%, \lambda=3.625, \theta=0.205, \sigma=0.965, r=6 \% . \sigma$ is the instantaneous volatility, $\theta$ the equilibrium mean level, and $\lambda$ the speed of reversion. The base parameters are taken by averaging the calibrated parameter values in Table 1 . 


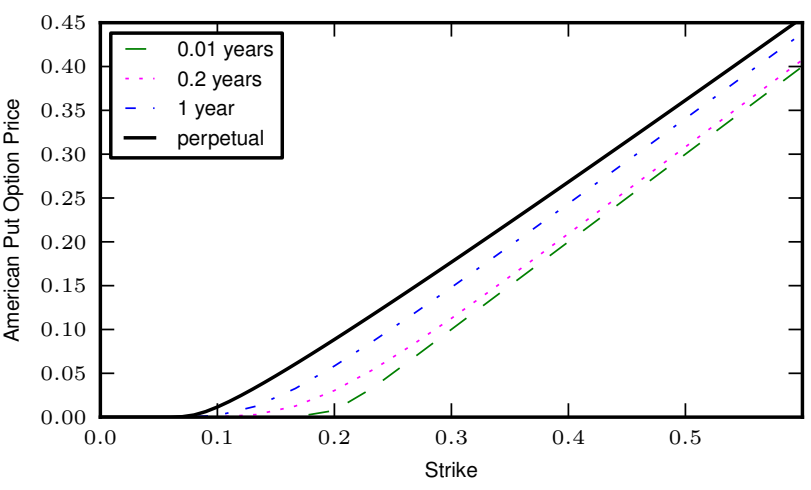

(a) American put option price

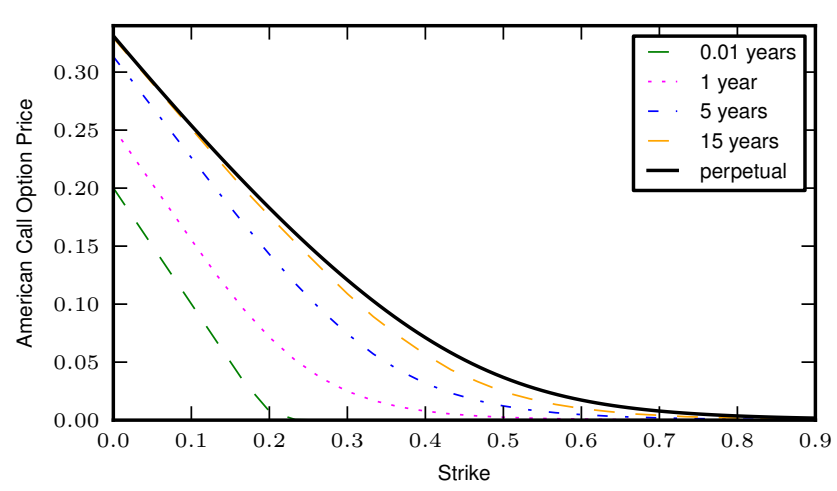

(b) American call option price

Figure 3

American options for different expiration times.

The base parameters are: $X_{0}=20 \%, \lambda=3.625, \theta=0.205, \sigma=0.965, r=6 \%$. $\sigma$ is the instantaneous volatility, $\theta$ the equilibrium mean level, and $\lambda$ the speed of reversion. 


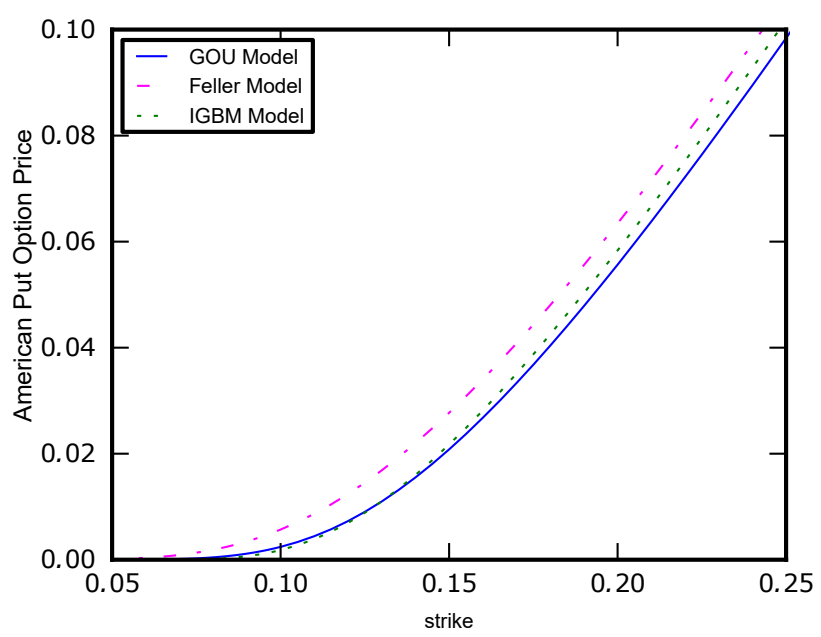

(a) American put option price

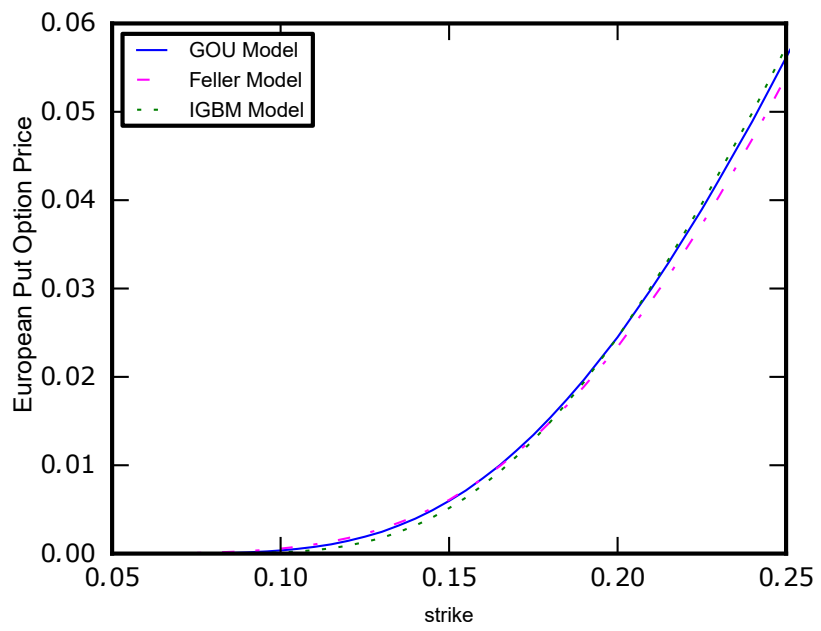

(c) European put option price

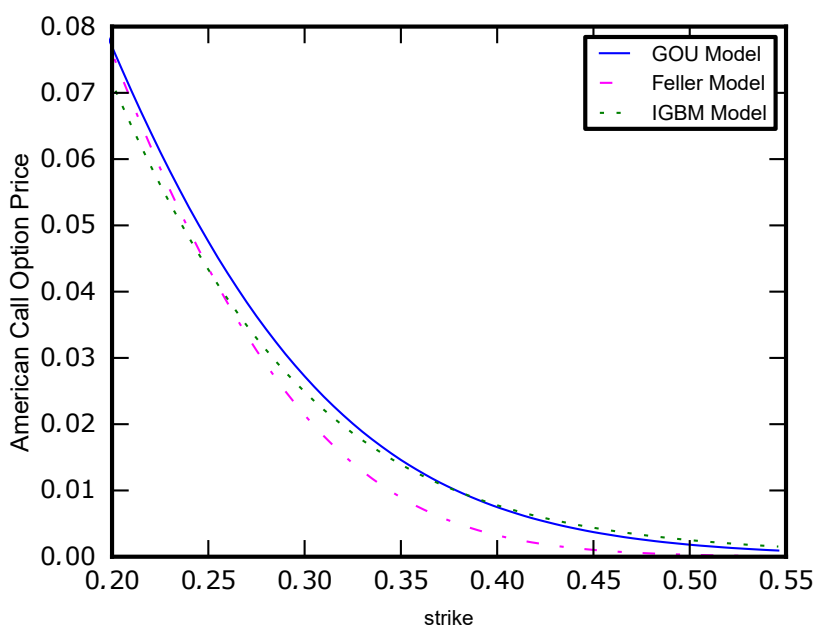

(b) American call option price

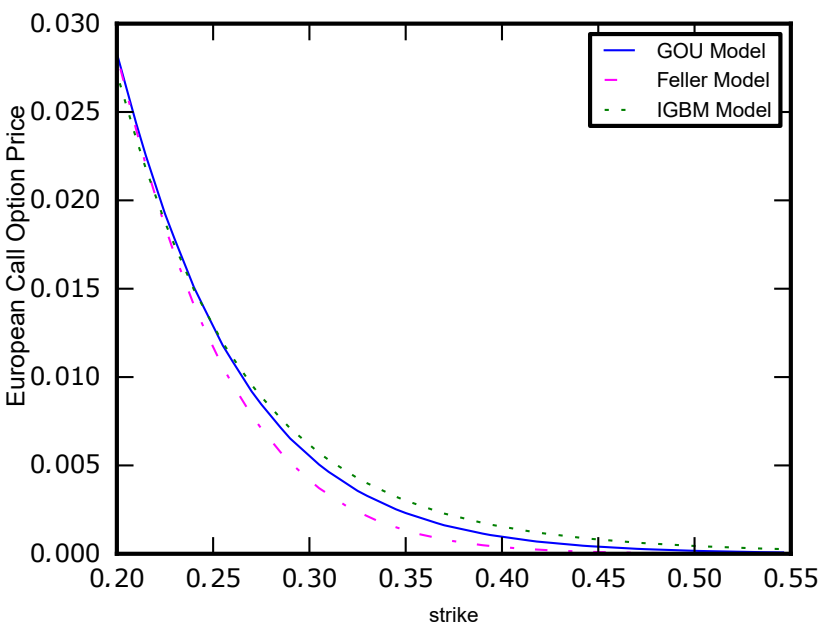

(d) European call option price

Figure 4

American options and European options for the three mean-reverting processes.

The base parameters are $T=1, r=6 \%, X_{0}=0.2$. For the GOU process, $\lambda=3.832, \theta=-1.651, \sigma=0.969$. For the Feller process, $\lambda=4.605, \theta=0.205, \sigma=0.469$. For the IGBM process, $\lambda=3.625, \theta=0.205, \sigma=0.965 . \sigma$ is the instantaneous volatility, $\theta$ the equilibrium mean level, and $\lambda$ the speed of reversion. 
Table 1

Calibrated parameter values for the three mean-reverting processes.

This table reports the calibrated parameter values for the three mean-reverting processes. In Panel A, parameters are estimated from stationary distributions and quadratic variations. In Panel B, parameters are estimated from the maximum likelihood method proposed in Aït-Sahalia (2002) and Yu (2007). In sub-period one, data start from January 2, 1990 to December 31, 2001. In sub-period two, data start from January 1, 2002 to September 30, 2012. $\sigma$ is the instantaneous volatility, $\theta$ the equilibrium mean level, and $\lambda$ the speed of reversion.

\begin{tabular}{|c|c|c|c|c|c|c|}
\hline & \multicolumn{3}{|c|}{ 1st sub-period } & \multicolumn{3}{|c|}{ 2nd sub-period } \\
\hline & GOU & Feller & IGBM & GOU & Feller & IGBM \\
\hline \multicolumn{7}{|l|}{ Panel A } \\
\hline$\lambda$ & 4.453 & 4.770 & 4.198 & 3.434 & 3.877 & 3.278 \\
\hline$\theta$ & -1.684 & 0.195 & 0.195 & -1.613 & 0.216 & 0.215 \\
\hline$\sigma$ & 0.911 & 0.415 & 0.911 & 1.023 & 0.519 & 1.023 \\
\hline Log likelihood & 4390.698 & 4353.171 & 4398.39 & 3073.601 & 2931.874 & 3149.607 \\
\hline \multicolumn{7}{|l|}{ Panel B } \\
\hline & 4.467 & 4.994 & 4.198 & 3.444 & 4.397 & 3.653 \\
\hline$\lambda$ & $(0.8731)$ & $(0.9210)$ & $(0.9264)$ & $(0.8079)$ & $(0.9030)$ & $(0.9346)$ \\
\hline O & -1.678 & 0.196 & 0.196 & -1.623 & 0.215 & 0.211 \\
\hline$\theta$ & $(0.0591)$ & $(0.0107)$ & $(0.0130)$ & $(0.0909)$ & $(0.0167)$ & $(0.0190)$ \\
\hline & 0.915 & 0.417 & 0.915 & 1.026 & 0.521 & 1.027 \\
\hline$\sigma$ & (0.0119) & $(0.0054)$ & (0.0119) & $(0.0140)$ & $(0.0071)$ & $(0.0141)$ \\
\hline Log likelihood & 9456.37 & 9292.71 & 9456.86 & 7956.18 & 7613.76 & 7958.10 \\
\hline
\end{tabular}


Table 2

Stationary state distributions of the three mean-reverting processes.

This table summarizes the steady-state distributions and quadratic variations of the three mean-reverting processes. In the steady-state column, $\kappa=\frac{2 \lambda}{\sigma^{2}}$. $\sigma$ is the instantaneous volatility, $\theta$ the equilibrium mean level, $\lambda$ the speed of reversion and $\left\{W_{t}\right\}_{t \geq 0}$ is a standard Wiener process.

\begin{tabular}{cccc}
\hline & Steady-State & Transformed Process & $\sigma^{2} T$ \\
\hline GOU & $\ln N\left(\theta, \frac{1}{\kappa}\right)$ & $d\left(\ln X_{t}\right)=\lambda\left(\theta-\ln X_{t}\right) d t+\sigma d W_{t}$ & $\sum_{i=0}^{T-1}\left(\ln X_{i+1}-\ln X_{i}\right)^{2}$ \\
Feller & $\frac{\gamma_{\kappa} \theta}{\kappa}$ & $d\left(2 \sqrt{X_{t}}\right)=\left(\frac{4 \lambda \theta-\sigma^{2}}{4 \sqrt{X_{t}}}-\lambda \sqrt{X_{t}}\right) d t+\sigma d W_{t}$ & $4 \sum_{i=0}^{T-1}\left(\sqrt{X_{i+1}}-\sqrt{X_{t}}\right)^{2}$ \\
IGBM & $\frac{\kappa \theta}{\gamma_{\kappa+1}}$ & $d\left(\ln X_{t}\right)=\left(\frac{\lambda \theta}{X_{t}}-\lambda-\frac{\sigma^{2}}{2}\right) d t+\sigma d W_{t}$ & $\sum_{i=0}^{T-1}\left(\ln X_{i+1}-\ln X_{i}\right)^{2}$ \\
\hline
\end{tabular}


Table 3

MFPT and the perpetual option values for put and call options.

This table reports the MFPT and the perpetual option values for several strikes and initial levels under the IGBM assumption using a reasonable set of parameter values as well as critical values. The base parameter values: $\lambda=3.625, \theta=0.205, \sigma=0.965, r=6 \%$. $\sigma$ is the instantaneous volatility, $\theta$ the equilibrium mean level, and $\lambda$ the speed of reversion.

\begin{tabular}{rrrrrrrrr}
\hline & \multicolumn{4}{c}{$X_{0}=15 \%$} & \multicolumn{2}{c}{$X_{0}=25 \%$} & \multicolumn{2}{c}{$X_{0}=40 \%$} \\
\hline \multirow{6}{*}{ Strike } & Threshold & MFPT & Price & MFPT & Price & MFPT & Price \\
& $(\%)$ & $(\%)$ & (years) & $(\%)$ & (years) & $(\%)$ & (years) & $(\%)$ \\
\hline \multirow{6}{*}{ Put } & 10 & 7.92 & 11.41 & 1.24 & 11.90 & 1.20 & 12.12 & 1.19 \\
& 15 & 8.95 & 4.11 & 4.87 & 4.60 & 4.73 & 4.82 & 4.67 \\
& 20 & 9.54 & 2.58 & 9.07 & 3.07 & 8.81 & 3.29 & 8.69 \\
& 25 & 9.99 & 1.90 & 13.49 & 2.39 & 13.11 & 2.61 & 12.93 \\
& 30 & 10.35 & 1.51 & 18.04 & 2.00 & 17.53 & 2.22 & 17.29 \\
& 40 & 10.94 & 1.06 & 27.35 & 1.55 & 26.57 & 1.77 & 26.21 \\
& 60 & 11.85 & 0.63 & 46.43 & 1.12 & 45.11 & 1.34 & 44.51 \\
\hline \multirow{6}{*}{ Call } & 10 & 45.30 & 6.15 & 25.67 & 5.59 & 26.53 & 2.63 & 31.16 \\
& 15 & 46.86 & 7.22 & 22.11 & 6.66 & 22.86 & 3.70 & 26.84 \\
& 20 & 48.61 & 8.62 & 18.74 & 8.05 & 19.37 & 5.10 & 22.75 \\
& 25 & 50.59 & 10.50 & 15.57 & 9.94 & 16.10 & 6.98 & 18.91 \\
& 30 & 52.84 & 13.11 & 12.66 & 12.54 & 13.09 & 9.59 & 15.37 \\
& 40 & 58.34 & 22.21 & 7.75 & 21.64 & 8.02 & 18.69 & 9.41 \\
& 60 & 74.06 & 89.63 & 2.15 & 89.07 & 2.22 & 86.11 & 2.61 \\
\hline
\end{tabular}


Table 4

Boundary types.

This table lists the boundary types of the three mean-reverting processes. $\sigma$ is the instantaneous volatility, $\theta$ the equilibrium mean level, $\lambda$ the speed of reversion and $\left\{W_{t}\right\}_{t \geq 0}$ is a standard Wiener process.

\begin{tabular}{cccc}
\hline & Dynamics & 0 & $\infty$ \\
\hline GOU & $d \ln X_{t}=\lambda\left(\theta-\ln X_{t}\right) d t+\sigma d W_{t}$ & Natural & Natural \\
Feller & $d X_{t}=\lambda\left(\theta-X_{t}\right) d t+\sigma \sqrt{X_{t}} d W_{t}$ & Entrance & Natural \\
IGBM & $d X_{t}=\lambda\left(\theta-X_{t}\right) d t+\sigma X_{t} d W_{t}$ & Entrance & Natural \\
\hline
\end{tabular}




\section{Appendix A. Ordinary Differential Equation (ODE)}

Lemma 1. Let $r>0$ and $\theta>0$, the general solution to $O D E$

$$
\lambda(\theta-x) \frac{d}{d x} V(x)+\frac{1}{2} \sigma^{2} x^{2} \frac{d^{2} V}{d x^{2}}=r V(x)
$$

is given by

$$
V(x)=A x^{-a} M\left(a, b ; \frac{c}{x}\right)+B x^{-a} U\left(a, b ; \frac{c}{x}\right)
$$

where

$$
a=\frac{\sqrt{\left(2 \lambda+\sigma^{2}\right)^{2}+8 r \sigma^{2}}-\left(2 \lambda+\sigma^{2}\right)}{2 \sigma^{2}}, \quad b=\frac{2 \lambda}{\sigma^{2}}+2 a+2, \quad c=\frac{2 \lambda \theta}{\sigma^{2}}
$$

and the coefficients $A, B \in \mathbb{R}$.

Proof. Let $V(x)=x^{\alpha} W\left(\frac{1}{x}\right)$. After rearranging, ODE A.1 becomes

$$
\begin{aligned}
& x^{\alpha} W\left(\frac{1}{x}\right)\left(\sigma^{2} \alpha(\alpha-1)-2 \lambda \alpha-2 r\right) \\
& +x^{\alpha-1}\left(\sigma^{2} \frac{1}{x} W^{\prime \prime}\left(\frac{1}{x}\right)+2\left[\lambda-(\alpha-1) \sigma^{2}-\lambda \theta \frac{1}{x}\right] W^{\prime}\left(\frac{1}{x}\right)+2 \lambda \theta \alpha W\left(\frac{1}{x}\right)\right)=0
\end{aligned}
$$

The root of the quadratic equation

$$
\sigma^{2} \alpha(\alpha-1)-2 \lambda \alpha-2 r=0
$$

is

$$
\alpha=\frac{\left(2 \lambda+\sigma^{2}\right) \pm \sqrt{\left(2 \lambda+\sigma^{2}\right)^{2}+8 r \sigma^{2}}}{2 \sigma^{2}}
$$

We choose

$$
\alpha=\frac{\left(2 \lambda+\sigma^{2}\right)-\sqrt{\left(2 \lambda+\sigma^{2}\right)^{2}+8 r \sigma^{2}}}{2 \sigma^{2}}<0 .
$$


Let $y=\frac{1}{x}$, we have

$$
\sigma^{2} y W^{\prime \prime}(y)+2\left[\lambda-(\alpha-1) \sigma^{2}-\lambda \theta y\right] W^{\prime}(y)+2 \lambda \theta \alpha W(y)=0
$$

Let $W(y)=U(w)$ where $w=\frac{2 \lambda \theta y}{\sigma^{2}}$, then

$$
w U^{\prime \prime}(w)+\left(\frac{2 \lambda}{\sigma^{2}}-2(\alpha-1)-w\right) U^{\prime}(w)+\alpha U(w)=0
$$

ODE (A.2) is the Kummer equation. It has two independent solutions $M$ and $U$ (see Appendix B). Therefore the general solution to ODE (A.1) is given by

$$
V(x)=A x^{\alpha} M\left(-\alpha, \frac{2 \lambda}{\sigma^{2}}-2(\alpha-1) ; \frac{2 \lambda \theta}{\sigma^{2} x}\right)+B x^{\alpha} U\left(-\alpha, \frac{2 \lambda}{\sigma^{2}}-2(\alpha-1) ; \frac{2 \lambda \theta}{\sigma^{2} x}\right)
$$




\section{Appendix B. Confluent Hypergeometric Functions}

The Ordinary Differential Equation (ODE)

$$
x \frac{d^{2} f}{d x^{2}}+(b-x) \frac{d f}{d x}-a f=0
$$

has two independent solutions $M(a, b ; x)$ and $U(a, b ; x)$. The solution

$$
M(a, b ; x)=1+\frac{a x}{b}+\frac{(a)_{2} x^{2}}{(b)_{2} 2 !}+\ldots+\frac{(a)_{n} x^{n}}{(b)_{n} n !}+\ldots
$$

where $(a)_{n}=\frac{\Gamma(a+n)}{\Gamma(a)}=a(a+1) \ldots(a+n-1)$ is the Pochhammer symbol with the convention $(a)_{0}=1$, is called the confluent hypergeometric function of first kind (also called the Kummer function). The solution

$$
U(a, b ; x)=\frac{\pi}{\sin \pi b}\left(\frac{M(a, b ; x)}{\Gamma(1+a-b) \Gamma(b)}-x^{1-b} \frac{M(1+a-b, 2-b ; x)}{\Gamma(a) \Gamma(2-b)}\right)
$$

is called the confluent hypergeometric function of second kind (also called the Tricomi function). The Tricomi is not defined everywhere. For details, we refer to Abramowitz and Stegun (1972).

The first order derivative of the Kummer function is

$$
\frac{d M(a, b ; x)}{d x}=\frac{a}{b} M(a+1, b+1 ; x)
$$

Asymptotic behaviors for the confluent hypergeometric functions $M$ and $U$ are as follows. When $|x| \rightarrow 0$

$$
\begin{gathered}
U(a, b ; x)=\frac{\Gamma(b-1)}{\Gamma(a)} x^{1-b}+O\left(|x|^{b-2}\right), \quad \text { as } \quad b>2 . \\
M(a, b ; 0)=1, \quad \text { as } \quad b \neq-n .
\end{gathered}
$$

When $|x| \rightarrow \infty$

$$
M(a, b ; x)=\frac{\Gamma(b)}{\Gamma(a)} e^{x} x^{a-b}\left(1+O\left(x^{-1}\right)\right), \quad \text { as } \quad x>0
$$




$$
\begin{gathered}
M(a, b, x)=\frac{\Gamma(b)}{\Gamma(b-a)}(-x)^{-a}\left(1+O\left((-x)^{-1}\right)\right), \quad \text { as } \quad x<0 . \\
U(a, b ; x)=x^{-a}\left(1+O\left(x^{-1}\right)\right), \quad \text { as } x>0 .
\end{gathered}
$$




\section{Appendix C. Proofs}

Lemma 2. For $v>u>0$ and $x \in \mathbb{R}, \frac{M(u, v+1 ; x)}{M(u, v ; x)}$ is a strictly decreasing function with respect to $x$.

Proof. The integral representation $M(u, v ; x)=\frac{\Gamma(v)}{\Gamma(v-u) \Gamma(u)} \int_{0}^{1} e^{x t} t^{u-1}(1-t)^{v-u-1} d t$ implies $0 \leq$ $M(u, v ; x), M(u, v+1 ; x)$. We have

$$
(\ln M(u, v+1 ; x)-\ln M(u, v ; x))^{\prime}=\frac{v-u}{v} \frac{M(u, v+1 ; x)}{M(u, v ; x)}-\frac{v+1-u}{v+1} \frac{M(u, v+2 ; x)}{M(u, v+1 ; x)}
$$

From the integral representation and the fact that $e^{x t} t^{u-1}(1-t)^{\nu-u-1}$ and $e^{x t} t^{u-1}(1-t)^{v-u+1}$ are not linearly dependent, we use the Cauchy-Schwarz inequality and get

$$
\frac{\Gamma^{2}(v+1-u) \Gamma^{2}(u)}{\Gamma^{2}(v+1)} M^{2}(u, v+1 ; x)<\frac{\Gamma(v+2-u) \Gamma(v-u) \Gamma^{2}(u)}{\Gamma(v+2) \Gamma(v)} M(u, v+2 ; x) M(u, v ; x)
$$

which reduces to

$$
\frac{v-u}{v} \frac{M(u, v+1 ; x)}{M(u, v ; x)}<\frac{v+1-u}{v+1} \frac{M(u, v+2 ; x)}{M(u, v+1 ; x)} .
$$

Therefore $\ln \frac{M(u, v+1 ; x)}{M(u, v ; x)}$ is strictly decreasing. The proof holds as $\ln (\cdot)$ is a strictly increasing function.

Proof of Proposition 1 Let $f(x)=\frac{c(b-a)}{b} \frac{M\left(a, b+1 ; \frac{c}{x}\right)}{M\left(a, b ; \frac{c}{x}\right)}$ and $g(x)=\frac{-(a+1) x^{2}+(a K-c) x+c K}{K-x}$. From

$$
g^{\prime}(x)=\frac{(a+1) x^{2}-2(a+1) K x+a K^{2}}{(K-x)^{2}}
$$

we see that $g(x)$ is strictly increasing when $0 \leq x<K \frac{\sqrt{a+1}-1}{\sqrt{a+1}}$ and strictly decreasing when $K \frac{\sqrt{a+1}-1}{\sqrt{a+1}} \leq x<K$. Since $b-a>0$, the continuous function $f(x)$ is strictly increasing by Lemma (2). Furthermore, by using the asymptotic expansions, we have

$$
\lim _{x \rightarrow \infty} f(x)=\frac{c(b-a)}{b}, \lim _{x \rightarrow 0+} f(x)=0 .
$$


And in addition

$$
\lim _{x \rightarrow 0} g(x)=c, \lim _{x \rightarrow K-} g(x)=-\infty
$$

Since $\frac{c(b-a)}{b}<c$, when $0 \leq x \leq K$, it yields that $f(x)$ and $g(x)$ intersect and they intersect only once. Moreover, the intersection point is somewhere on $\left(K \frac{\sqrt{a+1}-1}{\sqrt{a+1}}, K\right)$.

Proposition 6. For $x \geq h$ and $n \in \mathbb{Z}^{+}$, we have

$$
\frac{\partial^{n} V(x)}{\partial x^{n}}=(-1)^{n} \frac{\Gamma(a+n)}{\Gamma(a)} \frac{M\left(a+n, b ; \frac{c}{x}\right)}{M\left(a, b ; \frac{c}{x}\right) x^{n}} V(x) .
$$

Proof. The proof is by induction on $n$. An easy computation shows

$$
\frac{\partial V(x)}{\partial x}=-\frac{a}{x} \frac{M\left(a+1, b ; \frac{c}{x}\right)}{M\left(a, b ; \frac{c}{x}\right)} V(x)
$$

Therefore, Equation (C.1) holds for $n=0$ and $n=1$. Assume it is true for $n \leq k$, we prove it is true for $n=k+1$. We have

$$
\begin{gathered}
\frac{\partial^{k} V(x)}{\partial x^{k}}=-\frac{a+k-1}{x} \frac{M\left(a+k, b ; \frac{c}{x}\right)}{M\left(a+k-1, b ; \frac{c}{x}\right)} \frac{\partial^{k-1} V(x)}{\partial x^{k-1}} . \\
\left(\frac{a+k-1}{x} \frac{M\left(a+k, b ; \frac{c}{x}\right)}{M\left(a+k-1, b ; \frac{c}{x}\right)}\right)^{\prime} \\
=\frac{(a+k-1)^{2}}{x^{2}} \frac{M^{2}\left(a+k, b ; \frac{c}{x}\right)}{M^{2}\left(a+k-1, b ; \frac{c}{x}\right)}-\frac{(a+k-1)(a+k)}{x^{2}} \frac{M\left(a+k+1, b ; \frac{c}{x}\right)}{M\left(a+k-1, b ; \frac{c}{x}\right)} .
\end{gathered}
$$

Taking the derivation of Equation (C.2) on both sides, we have

$$
\frac{\partial^{k+1} V(x)}{\partial x^{k+1}}=(-1)^{k+1} \frac{\Gamma(a+k+1)}{\Gamma(a)} \frac{M\left(a+k+1, b ; \frac{c}{x}\right)}{M\left(a, b ; \frac{c}{x}\right) x^{k+1}} V(x)
$$

Lemma 3. For $u, v>0$ and $x \in \mathbb{R}, \frac{U(u, v+1 ; x)}{U(u, v ; x)}$ is strictly decreasing at $x$.

Proof. The integral representation $U(u, v ; x)=\frac{1}{\Gamma(u)} \int_{0}^{\infty} e^{-x t} t^{u-1}(1+t)^{v-u-1} d t$ implies $0 \leq$ $U(u, v ; x)<U(u, v+1 ; x)$. Using the derivative relation $U(u, v ; x)=U(u, v ; x)-U(u, v+1 ; x)$, 
we have

$$
(\ln U(u, v+1 ; x)-\ln U(u, v ; x))^{\prime}=\frac{U(u, v+1 ; x)}{U(u, v ; x)}-\frac{U(u, v+2 ; x)}{U(u, v+1 ; x)}
$$

From the integral representation and the fact that $e^{-x t} t^{u-1}(1+t)^{v-u-1}$ and $e^{-x t} t^{u-1}(1+t)^{\nu-u+1}$ are not linearly dependent, after using the Cauchy-Schwarz inequality, we have

$$
U^{2}(u, v+1 ; x)<U(u, v+2 ; x) U(u, v ; x)
$$

which implies $\ln \frac{U(u, v+1 ; x)}{U(u, v ; x)}$ is strictly decreasing. The proof holds as $\ln (\cdot)$ is a strictly increasing function.

Proof of Proposition 2 Let $f(x)=\frac{a}{x} \frac{U\left(a+1, b+1 ; \frac{c}{x}\right)}{U\left(a, b ; \frac{c}{x}\right)}$ and $g(x)=\frac{(a+1) x-a K}{c(x-K)}$, then $g^{\prime}(x)=-\frac{K}{c(x-K)^{2}}$ which implies $g(x)$ is a decreasing function when $x \geq K$. By the recursive relation

$$
\begin{gathered}
x U(u, v+1 ; x)=(v-u) U(u, v ; x)+U(u-1, v ; x) \\
u U(u+1, v ; x)=U(u, v ; x)-U(u, v-1 ; x),
\end{gathered}
$$

we have

$$
\begin{aligned}
f(x) & =\frac{a}{x} \frac{U\left(a+1, b+1 ; \frac{c}{x}\right)}{U\left(a, b ; \frac{c}{x}\right)} \\
& =\frac{a(b-a-1) U\left(a+1, b ; \frac{c}{x}\right)+a U\left(a, b ; \frac{c}{x}\right)}{c U\left(a, b ; \frac{c}{x}\right)}=\frac{a(b-a-1) U\left(a+1, b ; \frac{c}{x}\right)}{c U\left(a, b ; \frac{c}{x}\right)}+\frac{a}{c} \\
& =\frac{(b-a-1)\left(U\left(a, b ; \frac{c}{x}\right)-U\left(a, b-1 ; \frac{c}{x}\right)\right)}{c U\left(a, b ; \frac{c}{x}\right)}+\frac{a}{c} \\
& =-\frac{(b-a-1)}{c} \frac{U\left(a, b-1 ; \frac{c}{x}\right)}{U\left(a, b ; \frac{c}{x}\right)}+\frac{b-1}{c} .
\end{aligned}
$$

Since $b-a-1>0, b-1>0$ and $\frac{U\left(a, b-1 ; \frac{c}{x}\right)}{U\left(a, b ; \frac{c}{x}\right)}$ is a strictly decreasing function with respect to $x$ by Lemma 3 , we see that $f(x)$ is a strictly increasing function. Furthermore, by using the 
asymptotic expansions of function $U$, we have

$$
\lim _{x \rightarrow 0+} f(x)=\frac{a}{c}, \lim _{x \rightarrow \infty} f(x)=\frac{b-1}{c} .
$$

In addition,

$$
\lim _{x \rightarrow k+} g(x)=\infty, \lim _{x \rightarrow \infty} g(x)=\frac{a+1}{c} .
$$

Since $\frac{a+1}{c}<\frac{c-1}{c}$, when $x \geq k, f(x)$ and $g(x)$ intersect and they intersect only once. On the other hand, the critical value $h$ is the value such that $g(h)<\frac{b-1}{c}$, which implies $h>\frac{b-a-1}{b-a-2} K$.

Proposition 7. For $0<x<h$ and $n \in \mathbb{Z}^{+}$, we have

$$
\frac{\partial^{n} V(x)}{\partial x^{n}}=\frac{\Gamma(a+n) \Gamma(b-a)}{\Gamma(a) \Gamma(b-a-n) x^{n}} \frac{U\left(a+n, b ; \frac{c}{x}\right)}{U\left(a, b ; \frac{c}{x}\right)} V(x) .
$$

Proof. The proof is by induction on $n$. An easy computation shows

$$
\frac{\partial V(x)}{\partial x}=\frac{a(b-a-1)}{x} \frac{U\left(a+1, b ; \frac{c}{x}\right)}{U\left(a, b ; \frac{c}{x}\right)} V(x) .
$$

Therefore, Equation (C.3) holds for $n=0$ and $n=1$. Assume it is true for $n \leq k$, we prove it is true for $n=k+1$. We have

$$
\begin{aligned}
& \quad \frac{\partial^{k} V(x)}{\partial x^{k}}=\frac{(a+k-1)(b-a-k)}{x} \frac{U\left(a+k, b ; \frac{c}{x}\right)}{U\left(a+k-1, b ; \frac{c}{x}\right)} \frac{\partial^{k-1} V(x)}{\partial x^{k-1}} . \\
& \left(\frac{(a+k-1)(b-a-k)}{x} \frac{U\left(a+k, b ; \frac{c}{x}\right)}{U\left(a+k-1, b ; \frac{c}{x}\right)}\right)^{\prime} \\
& =-\frac{(a+k-1)^{2}(b-a-k)^{2}}{x^{2}} \frac{U^{2}\left(a+k, b ; \frac{c}{x}\right)}{U^{2}\left(a+k-1, b ; \frac{c}{x}\right)} \\
& +\frac{(a+k-1)(a+k)(b-a-k)(b-a-k-1)}{x^{2}} \frac{U\left(a+k+1, b ; \frac{c}{x}\right)}{U\left(a+k-1, b ; \frac{c}{x}\right)} .
\end{aligned}
$$

Taking the derivation of Equation (C.4) on both sides, we have

$$
\frac{\partial^{k+1} V(x)}{\partial x^{k+1}}=\frac{\Gamma(a+k+1) \Gamma(b-a)}{\Gamma(a) \Gamma(b-a-k-1) x^{k+1}} \frac{U\left(a+k+1, b ; \frac{c}{x}\right)}{U\left(a, b ; \frac{c}{x}\right)} V(x)
$$




\section{Proof of Proposition 5 . Critical value for perpetual put options:}

1. As $\lambda \rightarrow 0+, a \rightarrow \frac{\sqrt{\sigma^{2}+8 r}-\sigma}{2 \sigma}, c \rightarrow 0, b$ and $h$ are bounded. From asymptotic expansions of the Kummer function, we have $M\left(a, b ; \frac{c}{h}\right) \rightarrow 1$ and $M\left(a, b+1 ; \frac{c}{h}\right) \rightarrow 1$, which yields $h \rightarrow \frac{a K}{a+1}=\frac{\sqrt{\sigma^{2}+8 r}-\sigma}{\sqrt{\sigma^{2}+8 r}+\sigma} K$ from solving Equation 11 .

2. As $\sigma \rightarrow \infty$, we have $a \rightarrow 0$ and $c \rightarrow 0$. Since $b>0$, we have $M\left(a, b ; \frac{c}{h}\right)=1$ and $M\left(a, b+1 ; \frac{c}{h}\right)=1$, which leads to $h \rightarrow 0$.

3. As $\theta \rightarrow 0+, c \rightarrow 0+$, both $a$ and $b$ are bounded away from 0 . From Proposition 1 , $h>K \frac{\sqrt{a+1}-1}{\sqrt{a+1}}$ is bounded away from 0 . Therefore, the left side of Equation $(11)$ is 0 . Solving equation $\frac{-(a+1) h^{2}+(a K-c) h+c K}{K-h}=0$ yields $h=\frac{a K}{a+1}$.

4. As $\theta \rightarrow \infty, c \rightarrow \infty$. From the asymptotic expansion of the Kummer function, $\frac{M\left(a, b+1, \frac{c}{h}\right)}{M\left(a, b, \frac{c}{h}\right)} \rightarrow$ $\frac{b h}{c}$, therefore, $h$ solves the quadratic equation

$$
(b-2 a-1) h^{2}+(2 a K-b K-c) h+c K=0 .
$$

The solution

$$
h=\frac{(b-2 a) K+c-\sqrt{(2 a K-b K+c)^{2}+4 c K}}{2(b-2 a-1)} \rightarrow K .
$$

5. When $\sigma \rightarrow 0$ or $\lambda \rightarrow \infty$, both $b$ and $z=\frac{c}{x}$ in $M(a, b ; z)$ become large. To analyze the asymptotic behavior, we use the result from López and Pagola (2010) who provide asymptotic expansions of confluent hypergeometric function $M(a, b ; z)$ (and $U(a, b ; z)$ ) for large $b$ and $z$. Let

$$
\alpha=\frac{b-a-1}{z}=\frac{x}{\theta}\left(1+\frac{(a+1) \sigma^{2}}{2 \lambda}\right) .
$$


The asymptotic analysis depends on the sign of

$$
z+a+1-b=\frac{c}{x}+a+1-b=\frac{2 \lambda \theta}{\sigma^{2} x}-\frac{2 \lambda}{\sigma^{2}}-a-1=\frac{2 \lambda}{\sigma^{2}}\left(\frac{\theta}{x}-1\right)-a-1
$$

i) If $\theta<x$, then $z+a+1-b<0$, López and Pagola (2010) show

$$
\begin{gathered}
M(a, b ; z)=\frac{\Gamma(b)}{\Gamma(b-a)} \frac{1}{(b-a-z-1)^{a}}\left(1+\mathscr{O}\left(\frac{1}{b}\right)\right), \\
U(a, b ; z)=\frac{1}{\Gamma(a)}\left(\frac{\alpha}{e}\right)^{\alpha z}(\alpha-1)^{a-1} \sqrt{\frac{2 \alpha \pi}{z}}\left(1+\mathscr{O}\left(\frac{1}{z}\right)\right) .
\end{gathered}
$$

Therefore,

$$
\begin{aligned}
\frac{M\left(a, b+1, \frac{c}{x}\right)}{M\left(a, b, \frac{c}{x}\right)} & \sim \frac{b}{b-a}\left(\frac{b-a-\frac{c}{x}-1}{b-a-\frac{c}{x}}\right)^{a} \\
& \sim \frac{b}{b-a}\left(1+a \ln \left(1-\frac{1}{b-a-\frac{c}{x}}\right)\right) \\
& \sim \frac{b}{b-a}\left(1-\frac{a}{b-a-\frac{c}{x}}\right), \\
\frac{U\left(a+1, b+1, \frac{c}{x}\right)}{U\left(a, b, \frac{c}{x}\right)} & \sim \frac{1}{a}\left(\frac{b-a-1}{\frac{c}{x}}-1\right) .
\end{aligned}
$$

ii) If $\theta>x$, then $z+a+1-b>0$, López and Pagola (2010) show

$$
\begin{gathered}
M(a, b, z)=\frac{\Gamma(b) e^{z}(1-\alpha)^{a-1}}{\Gamma(a) \Gamma(b-a)}\left(\frac{\alpha}{e}\right)^{\alpha z} \sqrt{\frac{2 \alpha \pi}{z}}\left(1+\mathscr{O}\left(\frac{1}{z}\right)\right), \\
U(a, b ; z)=\frac{1}{(z+a+1-b)^{a}}\left(1+\mathscr{O}\left(\frac{1}{b}\right)\right) .
\end{gathered}
$$

Therefore,

$$
\begin{aligned}
\frac{M\left(a, b+1, \frac{c}{x}\right)}{M\left(a, b, \frac{c}{x}\right)} & \sim \frac{(b-a) x}{c} \\
\frac{U\left(a+1, b+1, \frac{c}{x}\right)}{U\left(a, b, \frac{c}{x}\right)} & \sim \frac{1}{\frac{c}{x}+a+1-b} .
\end{aligned}
$$


A) If $K<\theta$, on the one hand, $\lim _{c \rightarrow \infty} g(x)=c$, for $x<K$ and $\lim _{x \rightarrow K-} g(x)=-\infty$.

On the other hand, from the asymptotic analysis of the Kummer function,

$$
\lim _{c \rightarrow \infty} f(x)=\lim _{c \rightarrow \infty} \frac{c(b-a)}{b} \frac{M\left(a, b+1, \frac{c}{x}\right)}{M\left(a, b, \frac{c}{x}\right)}=\frac{c x}{\theta}<c, \quad \text { for } x<\theta
$$

Thus, the intersection point must be $h=K$.

B) If $\theta<K$, from the asymptotic analysis, we have

$$
\lim _{b \rightarrow \infty} f(x)=\left\{\begin{array}{cl}
\frac{(b-a)^{2}}{b} x, & x \leq \theta ; \\
c\left(1-\frac{a}{b-a-\frac{c}{x}}\right), & \theta<x<K .
\end{array}\right.
$$

The critical barrier $h$, thus, solves the quadratic equation

$$
c\left(1-\frac{a}{b-a-\frac{c}{x}}\right)=\frac{-(a+1) x^{2}+(a K-c) x+c K}{K-x},
$$

which yields

$$
h \sim \frac{c+a b K-a^{2} K}{(b-a)(a+1)} .
$$

As $\lambda \rightarrow \infty$, it is seen that $a \sim \frac{r}{\lambda}, \lim _{\lambda \rightarrow \infty} a b=\frac{2 r}{\sigma^{2}}$, which gives $\lim _{\lambda \rightarrow \infty} h=\theta$.

As $\sigma \rightarrow 0$, it is seen that $a \rightarrow \frac{r}{\lambda}, \lim _{\sigma \rightarrow 0} h=\frac{\theta+a K}{a+1}=\frac{\lambda \theta+r K}{r+\lambda}$.

Critical value for perpetual call options: The recurrence relation

$$
(b-a-1) U(a+1, b ; z)+U(a, b ; z)=z U(a+1, b+1 ; z)
$$

implies

$$
\begin{aligned}
& \frac{(b-a-1) U(a+1, b ; z)}{U(a+1, b+1 ; z)}+\frac{U(a, b ; z)}{U(a+1, b+1 ; z)}=z, \\
& \frac{(b-a-1) U\left(a+1, b ; \frac{c}{x}\right)}{U\left(a+1, b+1 ; \frac{c}{x}\right)}+\frac{a c(x-K)}{(a+1) x^{2}-a K x}=\frac{c}{x} .
\end{aligned}
$$


If $c \rightarrow 0+$, from the asymptotic expansion (B.1), the critical barrier $h$ solves

$$
\frac{(b-a-1) c}{(b-1) x}+\frac{a c(x-K)}{(a+1) x^{2}-a K x}=\frac{c}{x},
$$

which gives

$$
h=\frac{b-a-1}{b-a-2} K
$$

1. As $\lambda \rightarrow 0+, a \rightarrow \frac{\sqrt{\sigma^{2}+8 r}-\sigma}{2 \sigma}, b>2$ and $c \rightarrow 0$. From Equation C.10 it leads to $h \rightarrow$ $\frac{(a+1) K}{a}=\frac{\sqrt{\sigma^{2}+8 r}+\sigma}{\sqrt{\sigma^{2}+8 r}-\sigma} K$.

2. As $\sigma \rightarrow \infty$, we have $a \rightarrow 0, c \rightarrow 0$ and $b \rightarrow 2$. From Equation (C.10), it leads to $h \rightarrow \infty$.

3. As $\theta \rightarrow 0+, \lim _{\theta \rightarrow 0+} c=0$. From Equation (C.10), it yields

$$
h=\frac{b-a-1}{b-a-2} K
$$

4. As $\theta \rightarrow \infty, c \rightarrow \infty$. From Equation (C.9) and the asymptotic expansion (B.5), the critical value $h$ solves

$$
(b-a-1)+\frac{a c(x-K)}{(a+1) x^{2}-a K x}=\frac{c}{x},
$$

which leads to

$$
h=\frac{a K}{a+1}+\frac{c}{(b-a-1)(a+1)} \rightarrow \infty
$$

5. When $\sigma \rightarrow 0$ or $\lambda \rightarrow \infty$, both $b$ and $z=\frac{c}{x}$ in $M(a, b ; z)$ become large. We use the results from asymptote (C.6) and asymptote (C.8)

A) If $\theta<\frac{b-a-1}{b-a-2} K$, as $c \rightarrow \infty$. On the one hand,

$$
g(x)=\frac{(a+1) x-a K}{c(x-K)} \rightarrow 0 \text { for } x>\frac{b-a-1}{b-a-2} K
$$


and $g(K+)=\infty$. On the other hand, when $x>\frac{b-a-1}{b-a-2} K>\theta$,

$$
f(x)=\frac{a}{x} \frac{U\left(a+1, b+1, \frac{c}{x}\right)}{U\left(a, b, \frac{c}{x}\right)} \sim\left(\frac{b-a-1}{c}-\frac{1}{x}\right)=\frac{1}{\theta}-\frac{1}{x}>0 .
$$

It implies the intersection point must be $h=K$.

B) If $\frac{b-a-1}{b-a-2} K<\theta$,

$$
\lim _{b \rightarrow \infty} f(x)= \begin{cases}\left(\frac{b-a-1}{c}-\frac{1}{x}\right), & x>\theta ; \\ \frac{a}{c+(a+1-b) x}, & \frac{b-a-1}{b-a-2} K<x<\theta .\end{cases}
$$

The critical barrier $h$ solves the quadratic equation

$$
\frac{a}{c+(a+1-b) x}=\frac{(a+1) x-a K}{c(x-K)}
$$

which yields

$$
h \sim \frac{c+(b-a-1) a K}{(b-a-1)(a+1)}
$$

As $\lambda \rightarrow \infty$, it is seen that $a \sim \frac{r}{\lambda}, \lim _{\lambda \rightarrow \infty} a b=\frac{2 r}{\sigma^{2}}$, which gives $\lim _{\lambda \rightarrow \infty} h=\frac{c}{b}=\theta$. As $\sigma \rightarrow 0$, it is seen that $a \rightarrow \frac{r}{\lambda}, \lim _{\sigma \rightarrow 0} h=\frac{c+a b K}{b a+b}=\frac{\lambda \theta+r K}{r+\lambda}$. 